\title{
THE METRIC PROJECTIONS ONTO CLOSED CONVEX CONES IN A HILBERT SPACE
}

\author{
YANQI QIU AND ZIPENG WANG
}

\begin{abstract}
We study the metric projection onto the closed convex cone in a real Hilbert space $\mathscr{H}$ generated by a sequence $\mathcal{V}=\left\{v_{n}\right\}_{n=0}^{\infty}$. The first main result of this paper provides a sufficient condition under which we can identify the closed convex cone generated by $\mathcal{V}$ with the following set:

$$
\mathcal{C}[[\mathcal{V}]]:=\left\{\sum_{n=0}^{\infty} a_{n} v_{n} \mid a_{n} \geq 0, \text { the series } \sum_{n=0}^{\infty} a_{n} v_{n} \text { converges in } \mathscr{H}\right\} .
$$

Then, by adapting classical results on general convex cones, we give a useful description of the metric projection of a vector onto $\mathcal{C}[[\mathcal{V}]]$. As applications, we obtain the best approximations of many concrete functions in $L^{2}([-1,1])$ by polynomials with non-negative coefficients.
\end{abstract}

\section{INTRODUCTION}

1.1. Closed convex cone generated by a sequence of vectors. Recall that a nonempty subset of a vector space over the field $\mathbb{R}$ of real numbers is a convex cone if it is closed under addition and closed under multiplication by non-negative scalars. It is a classical problem to find the best approximation of a given vector by elements in a given closed convex subset, cf. $[5,7,8,10,12,14,16]$. The best approximation of functions by polynomials with non-negative coefficients on certain interval is particularly interesting, for instance, it plays a crucial role in the spectral analysis of self-adjoint operators on real Hilbert spaces $[12,14]$.

In this paper, we are interested in the convex cone generated by a given sequence in a real Hilbert space. More precisely, let $\mathscr{H}$ be a Hilbert space over $\mathbb{R}$ and let $\mathcal{V}=\left\{v_{n}\right\}_{n=0}^{\infty}$ be a sequence in $\mathscr{H}$. The convex cone $\mathcal{C}[\mathcal{V}]$ generated by the sequence $\mathcal{V}$ is the set of all non-negative linear combinations of the vectors in $\mathcal{V}$ (here and after, we denote by $\mathbb{N}$ the set of all non-negative integers: $\mathbb{N}=\{0,1,2, \cdots\})$ :

$$
\mathcal{C}[\mathcal{V}]:=\left\{\sum_{n=0}^{N} a_{n} v_{n} \mid N \in \mathbb{N}, a_{n} \geq 0\right\} .
$$

Denote by $\overline{\mathcal{C}}[\mathcal{V}]$ the closure of $\mathcal{C}[\mathcal{V}]$ inside $\mathscr{H}$. Then $\overline{\mathcal{C}}[\mathcal{V}]$ is again a convex cone and moreover is closed. The convex cone $\mathcal{C}[\mathcal{V}]$ and its closure $\overline{\mathcal{C}}[\mathcal{V}]$ are useful objects in functional analysis, mathematical optimization and many other fields, cf. [1, 2, 8, 13].

2010 Mathematics Subject Classification. Primary 52A27; Secondary 41A10, 46C05.

Key words and phrases. Closed convex cones; metric projections; best approximation; polynomials with non-negative coefficients.

Y. Qiu is supported by grants NSFC Y7116335K1, NSFC 11801547 and NSFC 11688101 of National Natural Science Foundation of China. Z. Wang is supported by NSFC 11601296 of National Natural Science Foundation of China. 
One can consult $[4,11]$ for the basic algebraic theory of $\mathcal{C}[\mathcal{V}]$ when $\mathcal{V}$ is a finite set of a finite dimensional Euclidean space and of an infinite dimensional Hilbert space respectively. Usually, it is more convenient to work with the closure $\overline{\mathcal{C}}[\mathcal{V}]$. In fact, the closedness of a convex subset is important in the best approximation theory [5, Chapter 5].

For any element $w \in \mathscr{H}$, let $d(w, \overline{\mathcal{C}}[\mathcal{V}])$ denote the distance of $w$ to $\overline{\mathcal{C}}[\mathcal{V}]$ :

$$
d(w, \overline{\mathcal{C}}[\mathcal{V}]):=\inf \{\|w-u\| \mid u \in \overline{\mathcal{C}}[\mathcal{V}]\}=\inf \left\{\left\|w-\sum_{n=0}^{N} a_{n} v_{n}\right\| \mid N \in \mathbb{N}, a_{n} \geq 0\right\} .
$$

For a non-zero vector $w \in \mathscr{H}$, we also introduce the relative distance $\lambda(w, \overline{\mathcal{C}}[\mathcal{V}])$ :

$$
\lambda(w, \overline{\mathcal{C}}[\mathcal{V}]):=\frac{d(w, \overline{\mathcal{C}}[\mathcal{V}])}{\|w\|} \in[0,1]
$$

Clearly, $\lambda(w, \overline{\mathcal{C}}[\mathcal{V}])=1$ if and only if $P_{\overline{\mathcal{C}}[\mathcal{V}]}(w)=0$, while $\lambda(w, \overline{\mathcal{C}}[\mathcal{V}])=0$ if and only if $w \in$ $\overline{\mathcal{C}}[\mathcal{V}]$. Denote by $\angle(w, v)$ the angle between two non-zero vectors $w, v$. If $\lambda(w, \overline{\mathcal{C}}[\mathcal{V}])<1$ then it is easy to see that it satisfies the equality

$$
\lambda(w, \overline{\mathcal{C}}[\mathcal{V}])=\sin (\inf \{\angle(w, v): v \in \overline{\mathcal{C}}[\mathcal{V}] \backslash\{0\}\})
$$

In other words, the quantity $\lambda(w, \overline{\mathcal{C}}[\mathcal{V}])$, when belongs to the open interval $(0,1)$, mesures how far the direction of the vector $w$ is away from the directions of the vectors in $\overline{\mathcal{C}}[\mathcal{V}]$.

By a classical result on closed convex subsets of Hilbert spaces (see e.g. [16, p. 239] and $\left[5\right.$, Theorem 3.5]), for any $w \in \mathscr{H}$, there exists a unique $w^{*} \in \overline{\mathcal{C}}[\mathcal{V}]$ closest to $w$ :

$$
\left\|w-w^{*}\right\|=d(w, \overline{\mathcal{C}}[\mathcal{V}])
$$

This unique element $w^{*}$ is called the metric projection of $w$ onto the closed convex cone $\overline{\mathcal{C}}[\mathcal{V}]$ and will be denoted by $P_{\overline{\mathcal{C}}[\mathcal{V}]}(w)$. In most situations, the computation of $d(w, \overline{\mathcal{C}}[\mathcal{V}])$ or $\lambda(w, \overline{\mathcal{C}}[\mathcal{V}])$ is then reduced to the characterization of $P_{\overline{\mathcal{C}}[\mathcal{V}]}(w)$.

For studying the best approximation of a given vector by elements in $\overline{\mathcal{C}}[\mathcal{V}]$, one may first try to understand better the closed convex cone $\overline{\mathcal{C}}[\mathcal{V}]$. However, in general, $\overline{\mathcal{C}}[\mathcal{V}]$ can be quite complicated. Therefore, in some particular cases, it seems to be of independent interests to find an explicit description of $\overline{\mathcal{C}}[\mathcal{V}]$. The first aim of this paper is to present an explicit description of $\overline{\mathcal{C}}[\mathcal{V}]$ under some additional assumptions on $\mathcal{V}$.

Let us introduce a subset $\mathcal{C}[[\mathcal{V}]]$ as follows:

$$
\mathcal{C}[[\mathcal{V}]]:=\left\{\sum_{n=0}^{\infty} a_{n} v_{n} \mid a_{n} \geq 0 \text { and the series } \sum_{n=0}^{\infty} a_{n} v_{n} \text { converges in } \mathscr{H}\right\} .
$$

We shall also denote $\mathcal{C}[[\mathcal{V}]]$ by $\mathcal{C}_{\mathscr{H}}[[\mathcal{V}]]$ when it is necessary. Clearly, we have

$$
\mathcal{C}[\mathcal{V}] \subset \mathcal{C}[[\mathcal{V}]] \subset \overline{\mathcal{C}}[\mathcal{V}]
$$

Remark. In general, the definition of $\mathcal{C}[[\mathcal{V}]]$ depends on the order of the vectors in the sequence $\mathcal{V}$. Indeed, the requirement that the series $\sum_{n=0}^{\infty} a_{n} v_{n}$ converges in $\mathscr{H}$ in general does not imply that it converges unconditionally and hence the limit may depend on the order. 
The subset $\mathcal{C}[[\mathcal{V}]]$ defined as above is again a convex cone and by (1.1), it is closed if and only if $\mathcal{C}[[\mathcal{V}]]=\overline{\mathcal{C}}[\mathcal{V}]$. Therefore, if $\mathcal{C}[[\mathcal{V}]]$ is closed, then we obtain a relatively simple description of $\overline{\mathcal{C}}[\mathcal{V}]$ : all elements of $\overline{\mathcal{C}}[\mathcal{V}]$ are given as a convergent series whose terms are multiples of elements in $\mathcal{V}$.

However, in general $\mathcal{C}[[\mathcal{V}]]$ is not closed. For instance, consider the two-dimensional Euclidean space $\mathbb{R}^{2}$ and let $v_{n}=(1, n) \in \mathbb{R}^{2}$ for $n \in \mathbb{N}$. Then $\overline{\mathcal{C}}[\mathcal{V}]=\{(x, y) \mid x, y \geq 0\}$. But $\mathcal{C}[[\mathcal{V}]] \neq \overline{\mathcal{C}}[\mathcal{V}]$ since $(0,1) \notin \mathcal{C}[[\mathcal{V}]]$

So we are going to investigate the following

Problem. When is $\mathcal{C}[[\mathcal{V}]]$ closed?

It is easy to see that $\mathcal{C}[[\mathcal{V}]]$ is closed if the sequence $\mathcal{V}$ satisfies the condition: there exist two constants $c, C>0$ and a sequence of positive numbers $\lambda_{n}>0$ such that the inequalities

$$
c\left(\sum_{n=0}^{\infty} \lambda_{n}\left|c_{n}\right|^{2}\right)^{1 / 2} \leq\left\|\sum_{n=0}^{\infty} c_{n} v_{n}\right\| \leq C\left(\sum_{n=0}^{\infty} \lambda_{n}\left|c_{n}\right|^{2}\right)^{1 / 2}
$$

hold for all finitely supported sequences $\left\{c_{n}\right\}_{n=0}^{\infty}$ in $\mathbb{R}$. A less obvious sufficient condition for $\mathcal{C}[[\mathcal{V}]]$ to be closed is given in the following Theorem 1.1.

Given a positive Radon measure $\mu$ on $\mathbb{R}_{+}=[0, \infty)$, we denote by $\operatorname{supp}(\mu)$ the topological support of $\mu$ and by $s_{\mu}$ the supremum of $\operatorname{supp}(\mu)$ :

$$
s_{\mu}:=\sup \{x \mid x \in \operatorname{supp}(\mu)\} \in[0, \infty] .
$$

We say that $\mu$ has finite moments of all orders if $\int_{\mathbb{R}_{+}} t^{n} d \mu(t)<\infty$ for all $n \in \mathbb{N}$.

Theorem 1.1. Let $\mu$ be a positive Radon measure on $\mathbb{R}_{+}$having finite moments of all orders and satisfying the condition:

$$
\mu\left(\mathbb{R}_{+} \backslash\left[0, s_{\mu}\right)\right)=0 .
$$

Let $\mathcal{W}=\left\{w_{n}\right\}_{n=0}^{\infty}$ be a sequence in another real Hilbert space $\mathscr{K}$ and assume that there exists a constant $C>0$ such that the inequality

$$
\left\|\sum_{n=0}^{\infty} a_{n} w_{n}\right\| \leq C\left\|\sum_{n=0}^{\infty} a_{n} t^{n}\right\|_{L^{2}(\mu)}
$$

holds for all finite supported sequences $\left\{a_{n}\right\}_{n=0}^{\infty}$ of non-negative real numbers. Assume that there exists a sequence $\left\{\lambda_{n}\right\}_{n=0}^{\infty}$ of positive numbers such that the sequence $\mathcal{V}=\left\{v_{n}\right\}_{n=0}^{\infty} \subset$ $\mathscr{H}$ satisfies

$$
\left\langle\lambda_{n} v_{n}, \lambda_{m} v_{m}\right\rangle_{\mathscr{H}}=\int_{\mathbb{R}} t^{m+n} d \mu(t)+\left\langle w_{n}, w_{m}\right\rangle_{\mathscr{K}} \quad \text { for all } n, m \in \mathbb{N} .
$$

Then we have $\overline{\mathcal{C}}[\mathcal{V}]=\mathcal{C}[[\mathcal{V}]]$ and thus $\mathcal{C}[[\mathcal{V}]]$ is a closed convex cone.

Remark. The condition $\mu\left(\mathbb{R}_{+} \backslash\left[0, s_{\mu}\right)\right)=0$ means that either $s_{\mu}=\infty$ or $\mu\left(\left\{s_{\mu}\right\}\right)=0$ if $s_{\mu}<\infty$. This condition in general can not be removed in Theorem 1.1. For instance, let $\nu$ be the Lebesgue measure on $[0,1]$ and let $\mu=\nu+\delta_{1}$, where $\delta_{1}$ is the Dirac mass at the point 1 , then the set

$$
\left\{\sum_{n=0}^{\infty} a_{n} t^{n} \mid a_{n} \geq 0, \text { the series } \sum_{n=0}^{\infty} a_{n} t^{n} \text { converges in } L^{2}(\mu)\right\}
$$


is not closed. Indeed, the sequence $\left\{t^{n}\right\}_{n=0}^{\infty}$ converges in $L^{2}(\mu)$ to the Dirac function $\delta_{1} \in L^{2}(\mu)$. But clearly, this limit function $\delta_{1}$ is not of the form $\sum_{n=0}^{\infty} a_{n} t^{n}$.

Remark. By modifying the proof of Theorem 1.1, we can replace the sequence of functions $\left\{t^{n}\right\}_{n=0}^{\infty}$ by any sequence $\left\{h_{n}(t)\right\}_{n=0}^{\infty}$ of continuous non-decreasing non-negative functions on $\mathbb{R}_{+}$satisfying the property:

$$
\sum_{n=0}^{\infty} \frac{h_{n}(t)}{h_{n}(s)}<\infty \text { for any pair }(s, t) \text { with } 0 \leq t<s .
$$

Theorem 1.1 has the following useful corollary. Before stating the corollary, let us note that if there exists a constant $C>0$ such that

$$
\left\langle w_{n}, w_{m}\right\rangle \leq C \int_{\mathbb{R}_{+}} t^{m+n} d \mu(t) \text { for all } m, n \in \mathbb{N},
$$

then the condition (1.2) is satisfied with the constant given by $\sqrt{C}$.

Corollary 1.2. Let $\nu$ be a positive Radon measure on $\mathbb{R}$. Assume that the restriction $\mu=\left.\nu\right|_{\mathbb{R}_{+}}$of the measure $\nu$ on $\mathbb{R}_{+}$has finite moments of all orders and satisfies the condition $\mu\left(\mathbb{R}_{+} \backslash\left[0, s_{\mu}\right)\right)=0$. Assume moreover that there exists a constant $C>0$ such that

$$
\int_{\mathbb{R}_{-}} t^{2 n} d \nu(t) \leq C \int_{\mathbb{R}_{+}} t^{2 n} d \nu(t) \quad \text { for all } n \in \mathbb{N}
$$

where $\mathbb{R}_{-}=\mathbb{R} \backslash \mathbb{R}_{+}$. Then

$$
\mathcal{C}_{L^{2}(\nu)}\left[\left[\left\{t^{n}\right\}_{n=0}^{\infty}\right]\right]=\left\{\sum_{n=0}^{\infty} a_{n} t^{n} \mid a_{n} \geq 0, \text { the series } \sum_{n=0}^{\infty} a_{n} t^{n} \text { converges in } L^{2}(\nu)\right\}
$$

is a closed convex cone in $L^{2}(\nu)$.

Remark. In general, the condition (1.4) can not be removed in Corollary 1.2. For instance, consider the Lebesgue measure on the interval $[-1,0]$ and the associated Hilbert space $L^{2}([-1,0])$. Then the set

$$
\mathcal{C}_{L^{2}([-1,0])}\left[\left[\left\{t^{n}\right\}_{n=0}^{\infty}\right]\right]=\left\{\sum_{n=0}^{\infty} a_{n} t^{n} \mid a_{n} \geq 0, \text { the series } \sum_{n=0}^{\infty} a_{n} t^{n} \text { converges in } L^{2}([-1,0])\right\}
$$

is not closed in $L^{2}([-1,0])$. See the Appendix of this paper for the details.

1.2. Metric projection onto a closed convex cone. Assume that the convex cone $\mathcal{C}[[\mathcal{V}]]$ is closed, that is $\mathcal{C}[[\mathcal{V}]]=\overline{\mathcal{C}}[\mathcal{V}]$. Proposition 1.3 below is an application to our situation of the classical results (cf. [16, Lemma 1.1]) on the metric projection onto a closed convex cone. We shall see that Proposition 1.3 can be useful in computing explicitly the metric projections of given vectors.

Definition. We say that a sequence $\mathcal{V}=\left\{v_{n}\right\}_{n=0}^{\infty}$ in a Hilbert space $\mathscr{H}$ has no positive relations, if the coincidence of two convergent series

$$
\sum_{n=0}^{\infty} a_{n} v_{n}=\sum_{n=0}^{\infty} b_{n} v_{n}
$$

with all coefficients non-negative implies $a_{n}=b_{n}$ for all $n \in \mathbb{N}$. 
Remark. Note that if the sequence $\mathcal{V}=\left\{v_{n}\right\}_{n=0}^{\infty}$ has no positive relations, then the vectors $v_{n}$ 's are linearly independent.

By convention, in what follows, we set

$$
\sum_{n \in \emptyset} a_{n} v_{n}:=0
$$

Proposition 1.3. Let $\mathcal{V}=\left\{v_{n}\right\}_{n=0}^{\infty} \subset \mathscr{H}$ be a sequence without positive relations and assume that $\mathcal{C}[[\mathcal{V}]]$ is closed. Then for any $w \in \mathscr{H}$, there exists a unique subset $S \subset \mathbb{N}$ such that

$$
P_{\mathcal{C}[\mathcal{V}]]}(w)=\sum_{n \in S} a_{n} v_{n} \quad \text { with } a_{n}>0 \text { for all } n \in S,
$$

where $\left(S,\left\{a_{n}\right\}_{n \in S}\right)$ is uniquely determined by

$$
\left\{\begin{array}{l}
\sum_{n \in S} a_{n} v_{n} \text { converges in } \mathscr{H} \text { and } a_{n}>0 \text { for all } n \in S \\
\sum_{k \in S} a_{k}\left\langle v_{k}, v_{n}\right\rangle \geq\left\langle w, v_{n}\right\rangle \text { for all } n \in \mathbb{N} \\
\sum_{k \in S} a_{k}\left\langle v_{k}, v_{n}\right\rangle=\left\langle w, v_{n}\right\rangle \text { for all } n \in S
\end{array}\right.
$$

Remark. Note that in Proposition 1.3, by saying that $\sum_{n \in S} a_{n} v_{n}$ converges in $\mathscr{H}$, we mean that the following limit exists in $\mathscr{H}$ :

$$
\lim _{N \rightarrow \infty} \sum_{n \in S, n \leq N} a_{n} v_{n}
$$

For any subset $S \subset \mathbb{N}$, define a subset $\mathscr{H}(\mathcal{V}, S) \subset \mathscr{H}$ by

$$
\mathscr{H}(\mathcal{V}, S):=\left\{w \in \mathscr{H} \mid P_{\mathcal{C}[[\mathcal{V}]]}(w)=\sum_{n \in S} a_{n} v_{n} \quad \text { with } a_{n}>0 \text { for all } n \in S\right\} .
$$

In particular, we have $\mathscr{H}(\mathcal{V}, \emptyset)=\left(P_{\mathcal{C}[[\mathcal{V}]]}\right)^{-1}(0)$.

By noting that the conditions in (1.6) are stable under addition and multiplication by a positive constant, we obtain the following corollary of Proposition 1.3.

Corollary 1.4. Let $\mathcal{V}=\left\{v_{n}\right\}_{n=0}^{\infty} \subset \mathscr{H}$ be a sequence without positive relations and assume that $\mathcal{C}[[\mathcal{V}]]$ is closed. Then we have a partition of the whole Hilbert space $\mathscr{H}$ :

$$
\mathscr{H}=\bigsqcup_{S \subset \mathbb{N}} \mathscr{H}(\mathcal{V}, S) .
$$

Moreover, for any subset $S \subset \mathbb{N}$, the subset $\{0\} \cup \mathscr{H}(\mathcal{V}, S)$ is a convex cone and the restriction of the metric projection

$$
\left.P_{\mathcal{C}[[\mathcal{V}]]}\right|_{\mathscr{H}(\mathcal{V}, S)}: \mathscr{H}(\mathcal{V}, S) \rightarrow \mathcal{C}[[\mathcal{V}]]
$$

is affine. That is, for any $\lambda_{1}, \lambda_{2}>0$ and any $w_{1}, w_{2} \in \mathscr{H}(\mathcal{V}, S)$, we have

$$
P_{\mathcal{C}[\mathcal{V}]]}\left(\lambda_{1} w_{1}+\lambda_{2} w_{2}\right)=\lambda_{1} P_{\mathcal{C}[[\mathcal{V}]]}\left(w_{1}\right)+\lambda_{2} P_{\mathcal{C}[[\mathcal{V}]]}\left(w_{2}\right)
$$


By considering the analogue of Proposition 1.3 for convex cone generated by finitely many vectors, we obtain in Corollary 1.5 a result for positive definite matrices. This result seems to be known in the litterature. We include it since we believe that our proof may be of its own interests.

Corollary 1.5. Assume that $A$ is a non-singular positive definite real-coefficient $n \times n$ matrix. Then for any $\left(c_{1}, \cdots, c_{n}\right) \in \mathbb{R}^{n}$, there exists a unique subset $S \subset\{1,2, \cdots, n\}$ and a unique $x \in \mathbb{R}^{S}$ such that

$$
\left\{\begin{array}{l}
x_{i}>0, \quad \text { for all } i \in S ; \\
\sum_{j \in S} a_{i j} x_{j}=c_{i}, \quad \text { for all } i \in S ; \\
\sum_{j \in S} a_{i j} x_{j} \geq c_{i}, \quad \text { for all } i \in\{1,2, \cdots, n\} .
\end{array}\right.
$$

Remark. If $c_{i} \leq 0$ for all $1 \leq i \leq n$, then we take $S=\emptyset$ in Corollary 1.5.

1.3. Computation of the metric projections. We shall see in $\S 1.4$ that Proposition 1.3 may be used to compute explicitly the metric projection of a vector onto the closed convex cone generated by a sequence. Note that our method is different from the one presented in [6].

The general scheme is given as follows (note that although we focus on the case of an infinite sequence $\mathcal{V}=\left\{v_{n}\right\}_{n=0}^{\infty}$, the same scheme is clearly still valid when the sequence is finite, that is $\mathcal{V}=\left\{v_{n}\right\}_{n=0}^{N}$ with $N \in \mathbb{N}$ ). The main assumptions for Proposition 1.3 are

(i) The sequence $\mathcal{V}=\left\{v_{n}\right\}_{n=0}^{\infty}$ has no positive relations.

(ii) The closed convex cone $\overline{\mathcal{C}}[\mathcal{V}]$ generated by the sequence $\mathcal{V}$ is given by $\overline{\mathcal{C}}[\mathcal{V}]=\mathcal{C}[[\mathcal{V}]]$. Under the above assumptions, assume that $w \in \mathscr{H}$ is a given vector and we want to compute the metric projection $P_{\mathcal{C}[[\mathcal{V}]]}(w)$. By Proposition 1.3, we shall and only need to determine a unique subset $S \subset \mathbb{N}$ (we will denote this subset by $S(w ; \mathcal{V}, \mathscr{H}$ ) if it is necessary) and a unique sequence $\left(a_{n}\right)_{n \in S}$ with $a_{n}>0$ for all $n \in S$ such that (1.6) is satisfied. For further reference, let us denote

$$
\Gamma(w ; \mathcal{V}, \mathscr{H}):=\left\{v_{n} \mid n \in S(w ; \mathcal{V}, \mathscr{H})\right\}
$$

The main difficulty in computing the metric projection $P_{\mathcal{C}[[\mathcal{V}]]}(w)$ is to determine the unique subset $S$, or equivalently, to determine the set $\Gamma(w ; \mathcal{V}, \mathscr{H})$. In general, it is not known to the authors whether there is an efficient way for determining such subset $S \subset \mathbb{N}$ for an arbitrarily given vector $w \in \mathscr{H}$. However, for a given vector $w \in \mathscr{H}$ and a given subset $S \subset \mathbb{N}$, by Proposition 1.3, it is relatively easier to determine whether the equality

$$
\Gamma(w ; \mathcal{V}, \mathscr{H})=\left\{v_{n} \mid n \in S\right\}
$$

holds or not (this is equivalent to determine whether $w \in \mathscr{H}(\mathcal{V}, S)$ or not, where $\mathscr{H}(\mathcal{V}, S)$ is defined as in (1.7)). Let us explain how to do so when $S \subset \mathbb{N}$ is a given finite subset. Set

$$
M:=\left(\left\langle v_{m}, v_{n}\right\rangle\right)_{m, n \in \mathbb{N}}
$$

and let $M_{S}$ be the sub-matrix indexed by $S \times S$ :

$$
M_{S}:=\left(\left\langle v_{m}, v_{n}\right\rangle\right)_{m, n \in S} .
$$


Then we need to solve the linear equation

$$
M_{S} x=y,
$$

where $x=\left(x_{n}\right)_{n \in S} \in \mathbb{R}^{S}$ is a column vector to be determined and $y \in \mathbb{R}^{S}$ is the column vector defined by $y=\left(\left\langle w, v_{n}\right\rangle\right)_{n \in S}$. The assumption that the sequence $\mathcal{V}$ has no positive relations implies that the matrix $M_{S}$ is non-singular (here we use the assumption that $S$ is finite) and thus the linear equation (1.9) has a unique solution, denoted by $\widehat{x} \in \mathbb{R}^{S}$. Now it remains to check whether the following conditions are satisfied:

$$
\left\{\begin{array}{cl}
\widehat{x}_{k}>0 & \text { for all } k \in S \\
\sum_{k \in S} \widehat{x}_{k}\left\langle v_{k}, v_{n}\right\rangle \geq\left\langle w, v_{n}\right\rangle & \text { for all } n \in \mathbb{N} \backslash S
\end{array} .\right.
$$

If (1.10) is satisfied, then $w \in \mathscr{H}(\mathcal{V}, S)$ and moreover, we obtain the desired metric projection $P_{\mathcal{C}[[\mathcal{V}]]}(w)$ :

$$
P_{\mathcal{C}[[\mathcal{V}]]}(w)=\sum_{k \in S} \widehat{x}_{k} v_{k}
$$

Otherwise, (1.10) is not satisfied, then $w \notin \mathscr{H}(\mathcal{V}, S)$ and we shall try other subsets $S \subset \mathbb{N}$ for computing $P_{\mathcal{C}[[\mathcal{V}]]}(w)$.

1.4. Applications in function theory. Consider the Lebesgue measure on $[-1,1]$ and the associated Hilbert space $L^{2}([-1,1])$. For easing the notation, set

$$
\mathcal{A}_{+}:=\mathcal{C}_{L^{2}([-1,1])}\left[\left[\left\{t^{n}\right\}_{n=0}^{\infty}\right]\right],
$$

that is,

$$
\mathcal{A}_{+}:=\left\{\sum_{n=0}^{\infty} a_{n} t^{n} \mid a_{n} \geq 0, \text { the series } \sum_{n=0}^{\infty} a_{n} t^{n} \text { converges in } L^{2}([-1,1])\right\} .
$$

By Corollary 1.2, the set $\mathcal{A}_{+}$is a closed convex cone in $L^{2}([-1,1])$. As before, the associated metric projection is denoted by $P_{\mathcal{A}_{+}}: L^{2}([-1,1]) \rightarrow \mathcal{A}_{+}$.

1.4.1. Power functions. For any $\beta \in(0, \infty)$, let $h_{\beta} \in L^{2}([-1,1])$ be the power function defined by

$$
h_{\beta}(t)=|t|^{\beta}, \quad t \in[-1,1] .
$$

It turns out that the best approximation of $h_{\beta}$ by elements in $\mathcal{A}_{+}$is given by a linear combination of two elements $t^{2 m}, t^{2 m+2}$ with $2 m$ and $2 m+2$ the closest two even numbers to $\beta$. For this reason, in what follows, it is convenient for us to write

$$
\beta=2 m+\alpha \quad \text { with } m \in \mathbb{N} \text { and } \alpha \in[0,2) .
$$

Proposition 1.6. Let $\alpha \in[0,2)$ and $m \in \mathbb{N}$. Then

$$
P_{\mathcal{A}_{+}}\left(h_{2 m+\alpha}\right)=a_{m} t^{2 m}+b_{m} t^{2 m+2},
$$

where $a_{m}$ and $b_{m}$ are given by

$$
\left\{\begin{array}{l}
a_{m}=a_{m}(\alpha):=\frac{(4 m+1)(4 m+3)}{(4 m+1+\alpha)(4 m+3+\alpha)} \frac{2-\alpha}{2}, \\
b_{m}=b_{m}(\alpha):=\frac{(4 m+3)(4 m+5)}{(4 m+1+\alpha)(4 m+3+\alpha)} \frac{\alpha}{2} .
\end{array}\right.
$$


Moreover, the distance $d\left(h_{2 m+\alpha}, \mathcal{A}_{+}\right)$is given by

$$
d\left(h_{2 m+\alpha}, \mathcal{A}_{+}\right)=\frac{\sqrt{2} \alpha(2-\alpha)}{(4 m+\alpha+1)(4 m+\alpha+3) \sqrt{4 m+2 \alpha+1}}
$$

and the relative distance $\lambda\left(h_{2 m+\alpha}, \mathcal{A}_{+}\right)$is given by

$$
\lambda\left(h_{2 m+\alpha}, \mathcal{A}_{+}\right)=\frac{\alpha(2-\alpha)}{(4 m+\alpha+1)(4 m+\alpha+3)} .
$$

Remark. Let $\alpha \in(0,2)$. Then the coefficients $a_{m}(\alpha)$ and $b_{m}(\alpha)$ in Proposition 1.3 satisfy

$$
a_{m}(\alpha)+b_{m}(\alpha)=\frac{(4 m+3)(4 m+2 \alpha+1)}{(4 m+1+\alpha)(4 m+3+\alpha)}>1 .
$$

Hence the best approximation in $\mathcal{A}_{+}$of the function $h_{2 m+\alpha}$ is not a convex combination of $t^{2 m}$ and $t^{2 m+2}$. In Figure 1, we draw the graphs in the first quadrant the best approximation in $\mathcal{A}_{+}$of $h_{3}$ :

$$
h_{3}(t)=|t|^{3}, \quad P_{\mathcal{A}_{+}}\left(h_{3}\right)=\frac{35}{96} t^{2}+\frac{21}{32} t^{4} .
$$

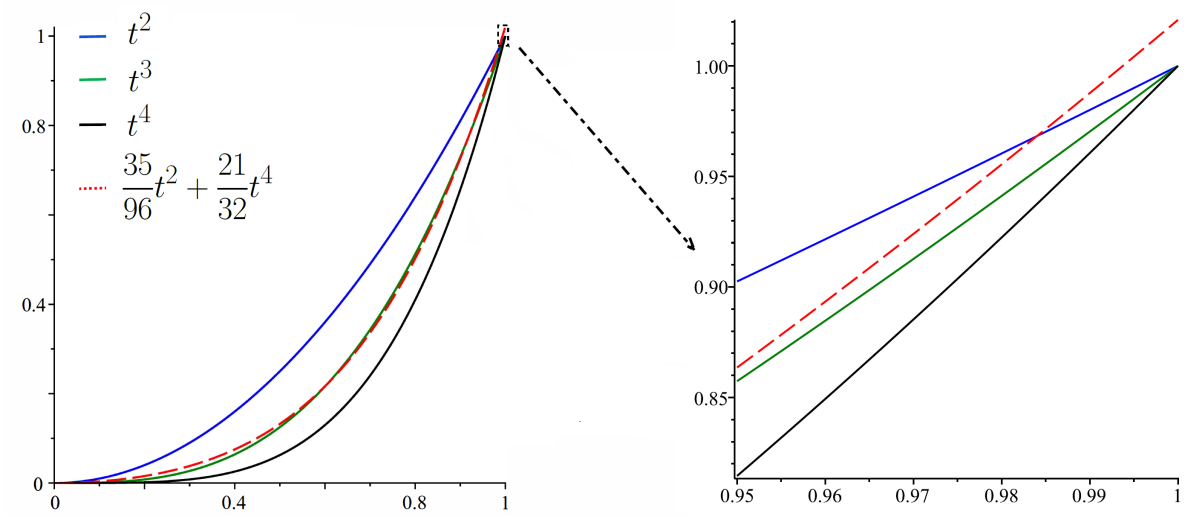

Figure 1. The best approximation of $|t|^{3}$ by polynomials with nonnegative coefficients: the graphs in the first quadrant.

Remark. Let $\alpha \in(0,2)$. Recall the definition (1.8). Proposition 1.3 implies the equality:

$$
\Gamma\left(|t|^{2 m+\alpha} ;\left\{t^{n}\right\}_{n=0}^{\infty}, L^{2}([-1,1])\right)=\left\{t^{2 m}, t^{4 m}\right\} .
$$

As we shall see in the proof of Proposition 1.3, it requires substantial efforts to prove the equality (1.14).

For explaining clearer the subtlety of the equality (1.14), let us consider the Hilbert space $L^{2}([0,1])$. It is easy to see that the equality $(1.14)$ is equivalent to the equality

$$
\Gamma\left(t^{2 m+\alpha} ;\left\{t^{2 n}\right\}_{n=0}^{\infty}, L^{2}([0,1])\right)=\left\{t^{2 m}, t^{4 m}\right\} .
$$

Note that the sequence $\left\{t^{n}\right\}_{n=0}^{\infty}$ now is repalced by the sequence $\left\{t^{2 n}\right\}_{n=0}^{\infty}$. That is, in the Hilbert space $L^{2}([0,1])$, the best approximation of the function $t^{2 m+\alpha}$ by elements in the closed convex cone generated by $\left\{t^{2 n}\right\}_{n=0}^{\infty}$ is given by a positive combination of the two functions $t^{2 m}$ and $t^{2 m+2}$. One may think that the equality (1.15) is a consequence 


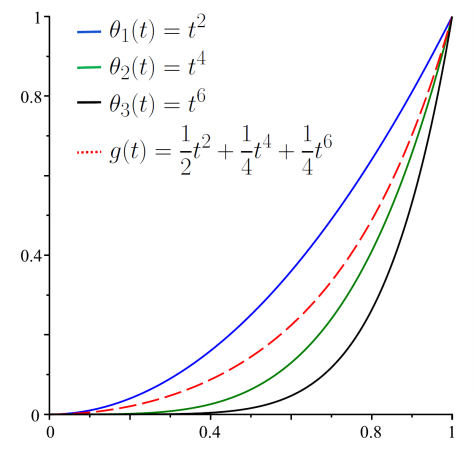

Figure 2. The best approximation in $\mathcal{C}\left(\theta_{1}, \theta_{2}, \theta_{3}\right)$ of the function $g$ is not given by positive combination of the two functions $\theta_{1}, \theta_{2}$, but is given by positive combination of all three functions $\theta_{1}, \theta_{2}, \theta_{3}$.

of the following observation: among the graphs of all functions $t^{0}, t^{2}, t^{4}, \cdots$ on $[0,1]$, the closest ones to that of the function $t^{2 m+\alpha}$ are exactly those of $t^{2 m}$ and $t^{2 m+2}$. However, let us point out that such kind of observation in general is not sufficient for deriving the equality (1.15). For instance, as shown in Figure 2, among the three graphs of $\theta_{1}, \theta_{2}, \theta_{3}$, the graphs of $\theta_{1}$ and $\theta_{2}$ are the closest ones to the graph of $g$. However, $g$ can be exactly approximated by elements in the convex cone $\mathcal{C}\left(\theta_{1}, \theta_{2}, \theta_{3}\right)$ generated by $\theta_{1}, \theta_{2}, \theta_{3}$ :

$$
g=\frac{1}{2} \theta_{1}+\frac{1}{4} \theta_{2}+\frac{1}{4} \theta_{3} \neq \lambda_{1} \theta_{1}+\lambda_{2} \theta_{2} \quad \text { for any } \lambda_{1} \geq 0, \lambda_{2} \geq 0
$$

hence we have

$$
\Gamma\left(g ;\left\{\theta_{1}, \theta_{2}, \theta_{3}\right\}, L^{2}([0,1])\right)=\left\{\theta_{1}, \theta_{2}, \theta_{3}\right\} \neq\left\{\theta_{1}, \theta_{2}\right\} .
$$

Corollary 1.7. For any $m \in \mathbb{N}$ and any positive Radon measure $\nu$ on $[0,2]$, we have

$$
P_{\mathcal{A}_{+}}\left(\int_{[0,2]} h_{2 m+\alpha} d \nu(\alpha)\right)=t^{2 m} \int_{[0,2]} a_{m}(\alpha) d \nu(\alpha)+t^{2 m+2} \int_{[0,2]} b_{m}(\alpha) d \nu(\alpha),
$$

where $a_{m}(\alpha), b_{m}(\alpha)$ are defined in (1.12).

Remark. The functions in Corollary 1.7 can be quite complicated: for instance, take $\nu$ the Lebesgue measure on $[0, \alpha]$ with $0<\alpha \leq 2$, then we have

$$
\int_{[0, \alpha]} h_{2 m+\alpha^{\prime}}(t) d \alpha^{\prime}=\frac{|t|^{2 m+\alpha}-t^{2 m}}{\log |t|} .
$$

1.4.2. Signed power functions. For any $\gamma \in(0, \infty)$, let $f_{\gamma} \in L^{2}([-1,1])$ be the signed power function defined by

$$
f_{\gamma}(t)=\operatorname{sgn}(t)|t|^{\gamma}, \quad t \in[-1,1] .
$$

Proposition 1.8. Let $\alpha \in[0,2)$ and $m \in \mathbb{N}$. Then

$$
P_{\mathcal{A}_{+}}\left(f_{2 m+1+\alpha}\right)=c_{m} t^{2 m+1}+d_{m} t^{2 m+3},
$$


where $c_{m}$ and $d_{m}$ are given by

$$
\left\{\begin{array}{l}
c_{m}=c_{m}(\alpha):=\frac{(4 m+3)(4 m+5)}{(4 m+3+\alpha)(4 m+5+\alpha)} \frac{2-\alpha}{2} \\
d_{m}=d_{m}(\alpha):=\frac{(4 m+5)(4 m+7)}{(4 m+3+\alpha)(4 m+5+\alpha)} \frac{\alpha}{2}
\end{array}\right.
$$

Moreover, the distance $d\left(f_{2 m+1+\alpha}, \mathcal{A}_{+}\right)$is given by

$$
d\left(f_{2 m+1+\alpha}, \mathcal{A}_{+}\right)=\frac{\sqrt{2} \alpha(2-\alpha)}{(4 m+\alpha+3)(4 m+\alpha+5) \sqrt{4 m+2 \alpha+3}}
$$

and the relative distance $\lambda\left(f_{2 m+1+\alpha}, \mathcal{A}_{+}\right)$is given by

$$
\lambda\left(f_{2 m+1+\alpha}, \mathcal{A}_{+}\right)=\frac{\alpha(2-\alpha)}{(4 m+\alpha+3)(4 m+\alpha+5)} .
$$

1.4.3. Indicator functions and non-decreasing functions. For any $a \in[-1,1)$, let $\psi_{a}$ be the function defined by

$$
\psi_{a}(t)=\mathbb{1}(t \geq a)=\left\{\begin{array}{ll}
0 & \text { if } t \in[-1, a) \\
1 & \text { if } t \in[a, 1]
\end{array} .\right.
$$

Recall the definition (1.8).

Proposition 1.9. Assume that $a \in[-1,1)$. Then the equality

$$
\Gamma\left(\psi_{a} ;\left\{t^{n}\right\}_{n=0}^{\infty}, L^{2}([-1,1])\right)=\left\{t^{n} \mid n=0,1,2\right\}
$$

holds if and only if

$$
0<a \leq \frac{1}{\sqrt{5}}
$$

Moreover, for any $a \in(0,1 / \sqrt{5}]$, the metric projection $P_{\mathcal{A}_{+}}\left(\psi_{a}\right)$ is given by

$$
P_{\mathcal{A}_{+}}\left(\psi_{a}\right)=\frac{1}{8}\left(4-9 a+5 a^{3}\right)+\frac{3}{4}\left(1-a^{2}\right) t+\frac{15}{8}\left(a-a^{3}\right) t^{2} .
$$

Proposition 1.10. Assume that $a \in[-1,1)$. Then the equality

$$
\Gamma\left(\psi_{a} ;\left\{t^{n}\right\}_{n=0}^{\infty}, L^{2}([-1,1])\right)=\left\{t^{n} \mid n=0,1\right\}
$$

holds if and only if

$$
-\frac{1}{\sqrt{5}} \leq a \leq 0
$$

Moreover, for any $a \in[-1 / \sqrt{5}, 0]$, the metric projection $P_{\mathcal{A}_{+}}\left(\psi_{a}\right)$ is given by

$$
P_{\mathcal{A}_{+}}\left(\psi_{a}\right)=\frac{1}{2}(1-a)+\frac{3}{4}\left(1-a^{2}\right) t
$$

Proposition 1.11. Assume that $a \in[-1,1)$. Then the equality

$$
\Gamma\left(\psi_{a} ;\left\{t^{n}\right\}_{n=0}^{\infty}, L^{2}([-1,1])\right)=\left\{t^{n} \mid n=0,1,2,3\right\}
$$

holds if and only if

$$
\frac{1}{\sqrt{5}}<a<\frac{\sqrt{105}-5}{10}
$$


Moreover, for any $a \in\left(\frac{1}{\sqrt{5}}, \frac{\sqrt{105}-5}{10}\right)$, the metric projection $P_{\mathcal{A}_{+}}\left(\psi_{a}\right)$ is given by

$$
\begin{gathered}
P_{\mathcal{A}_{+}}\left(\psi_{a}\right)=\frac{1}{8}(1-a)\left(4-5 a-5 a^{2}\right)+\frac{15}{32}\left(1-a^{2}\right)\left(3-7 a^{2}\right) t \\
+\frac{15}{8} a\left(1-a^{2}\right) t^{2}+\frac{35}{32}\left(1-a^{2}\right)\left(5 a^{2}-1\right) t^{3} .
\end{gathered}
$$

Remark. Propositions 1.9 and 1.11 may lead one to guess that there exists a sequence of critical points $\left\{b_{k}\right\}_{k=0}^{\infty}$ with $0<b_{0}<b_{1}<\cdots<b_{k}<\cdots<1$ such that

$$
\Gamma\left(\psi_{a} ;\left\{t^{n}\right\}_{n=0}^{\infty}, L^{2}([-1,1])\right)=\left\{t^{n} \mid n=0,1,2, \cdots, k+1, k+2\right\} \quad \text { for all } a \in\left(b_{k-1}, b_{k}\right) .
$$

This is however not clear to the authors at the time of writing. Indeed, the situation becomes more involved when $a$ is close to 1 . On the other hand, for negative $a$, the situation seems to be different. By [15], since $\mathcal{A}_{+}$is a closed convex cone in a Hilbert space, the metric projection $P_{A_{+}}: L^{2}([-1,1]) \rightarrow \mathcal{A}_{+}$is a continuous map. Therefore, by the formula (1.21), there exists $\varepsilon>0$ such that

$$
\{0,1\} \subset \Gamma\left(\psi_{a} ;\left\{t^{n}\right\}_{n=0}^{\infty}, L^{2}([-1,1])\right) \text { for all } a \in\left(-\frac{1}{\sqrt{5}}-\varepsilon,-\frac{1}{\sqrt{5}}\right) .
$$

But by Propositions 1.9, 1.10 and 1.11, for any $a \in\left(-\frac{1}{\sqrt{5}}-\varepsilon,-\frac{1}{\sqrt{5}}\right)$, we know that the set $\Gamma\left(\psi_{a} ;\left\{t^{n}\right\}_{n=0}^{\infty}, L^{2}([-1,1])\right)$ can not be any one of the three sets: $\{0,1\},\{0,1,2\}$ or $\{0,1,2,3\}$.

Propositions 1.9, 1.10 and 1.11 allow us to compute the metric projections onto the closed convex cone $\mathcal{A}_{+}$for functions in three large classes respectively. Let us state the consequence of Proposition 1.9 in Corollary 1.12 below, the consequences of Propositions 1.10 and 1.11 are similar and will be omitted.

Let $\mathcal{M}_{[0,1 / \sqrt{5}]}$ denote the class of functions on $[-1,1]$ consisting of all non-decreasing right continuous non-negative functions $\varphi:[-1,1] \rightarrow \mathbb{R}_{+}$such that

$$
\left.\varphi\right|_{[-1,0)} \equiv 0 \text { and }\left.\varphi\right|_{[1 / \sqrt{5}, 1]} \equiv \text { constant. }
$$

Note that any $\varphi \in \mathcal{M}_{[0,1 / \sqrt{5}]}$ uniquely determines a non-negative Radon measure, denoted by $d \varphi$, on the interval $[-1,1]$, by the formula

$$
d \varphi([-1, t]):=\varphi(t) \quad \text { for any } t \in[-1,1] .
$$

Moreover, the support $\operatorname{supp}(d \varphi)$ of the Radon measure $d \varphi$ satisfies $\operatorname{supp}(d \varphi) \subset[0,1 / \sqrt{5}]$ and we have

$$
\varphi(t)=\int_{[0,1 / \sqrt{5}]} \psi_{a}(t) d \varphi(a)=\int_{[0,1 / \sqrt{5}]} \mathbb{1}(t \geq a) d \varphi(a), \quad t \in[-1,1] .
$$

On the other hand, for any Radon measure on $[-1,1]$ with $\operatorname{support} \operatorname{supp}(\nu) \subset[0,1 / \sqrt{5}]$, the function $\varphi_{\nu}$, defined by the formula

$$
\varphi_{\nu}(t):=\nu([-1, t]) \quad \text { for all } t \in[-1,1],
$$

belongs to the class $\mathcal{M}_{[0,1 / \sqrt{5}]}$. 
Corollary 1.12. For any function $\varphi \in \mathcal{M}_{[0,1 / \sqrt{5}]}$, we have

$$
P_{\mathcal{A}_{+}}(\varphi)=A_{\varphi}+B_{\varphi} t+C_{\varphi} t^{2}
$$

with

$$
\left\{\begin{array}{l}
A_{\varphi}=\int_{[0,1 / \sqrt{5}]} \frac{1}{8}\left(4-9 a+5 a^{3}\right) d \varphi(a) \\
B_{\varphi}=\int_{[0,1 / \sqrt{5}]} \frac{3}{4}\left(1-a^{2}\right) d \varphi(a) \\
C_{\varphi}=\int_{[0,1 / \sqrt{5}]} \frac{15}{8}\left(a-a^{3}\right) d \varphi(a)
\end{array}\right.
$$

\section{Closedness of Convex Cones}

In this section, we prove Theorem 1.1 and Corollary 1.2.

Proof of Theorem 1.1. Let $\mathscr{H}_{\mathcal{V}} \subset \mathscr{H}$ denote the closed linear span of vectors in $\mathcal{V}$ :

$$
\mathscr{H}_{\mathcal{V}}:=\overline{\operatorname{span}}(\mathcal{V}) \subset \mathscr{H} \text {. }
$$

Let $\mathscr{H}(\mu, \mathcal{W})$ denote the closed linear span of the sequence $\left\{\left(t^{n}, w_{n}\right)\right\}_{n=0}^{\infty}$ in the real Hilbert space $L^{2}(\mu) \oplus \mathscr{K}$ :

$$
\mathscr{H}(\mu, \mathcal{W}):=\overline{\operatorname{span}}\left\{\left(t^{n}, w_{n}\right) \mid n \in \mathbb{N}\right\} \subset L^{2}(\mu) \oplus \mathscr{K} .
$$

The equalities (1.3) imply that the map $\left(t^{n}, w_{n}\right) \mapsto \lambda_{n} v_{n}$ can be extended to a linear isometric isomorphism between $\mathscr{H}(\mu, \mathcal{W})$ and $\mathscr{H}$. To complete the proof of Theorem 1.1, we shall prove that the following set

$$
\mathcal{C}(\mu, \mathcal{W}):=\left\{\sum_{n=0}^{\infty} a_{n}\left(t^{n}, w_{n}\right) \mid a_{n} \geq 0, \text { the series } \sum_{n=0}^{\infty} a_{n}\left(t^{n}, w_{n}\right) \text { converges in } L^{2}(\mu) \oplus \mathscr{K}\right\}
$$

is closed in $L^{2}(\mu) \oplus \mathscr{K}$.

Note first that for any element $(f, u) \in \mathcal{C}(\mu, \mathcal{W}) \subset L^{2}(\mu) \oplus \mathscr{K}$ with

$$
f=\sum_{n=0}^{\infty} a_{n} t^{n}, \quad a_{n} \geq 0,
$$

where the series converges in $L^{2}(\mu)$ and the equality is understood as elements in $L^{2}(\mu)$, there exists a subsequence $\left\{N_{k}\right\}_{k=0}^{\infty}$ of positive integers with $0<N_{0}<N_{1}<\cdots$ such that

$$
\lim _{k \rightarrow \infty} \sum_{n=0}^{N_{k}} a_{n} t^{n}=f(t)<\infty \text { for } \mu \text {-a.e. } t \in\left[0, s_{\mu}\right)
$$

By the assumption $a_{n} \geq 0$ for all $n \in \mathbb{N}$, for any $t_{0} \in\left[0, s_{\mu}\right)$ such that the limit equality (2.26) holds, we have

$$
\lim _{k \rightarrow \infty} \sum_{n=0}^{N_{k}} a_{n} t_{0}^{n}=\sum_{n=0}^{\infty} a_{n} t_{0}^{n}:=\lim _{N \rightarrow \infty} \sum_{n=0}^{N} a_{n} t_{0}^{n}
$$


It follows that we have the following $\mu$-almost everywhere equality:

$$
f(t)=\sum_{n=0}^{\infty} a_{n} t^{n}<\infty \quad \text { for } \mu \text {-a.e. } t \in\left[0, s_{\mu}\right) .
$$

By the definition of $s_{\mu}$ and the assumption $a_{n} \geq 0$ for all $n \in \mathbb{N}$, we even have

$$
\sum_{n=0}^{\infty} a_{n} t^{n}<\infty \quad \text { for all } t \in\left[0, s_{\mu}\right) .
$$

Now let $\left\{\left(f_{k}, u_{k}\right)\right\}_{k=0}^{\infty}$ be a sequence in $\mathcal{C}(\mu, \mathcal{W})$ :

$$
\left(f_{k}, u_{k}\right)=\left(\sum_{n=0}^{\infty} a_{n}^{(k)} t^{n}, \sum_{n=0}^{\infty} a_{n}^{(k)} w_{n}\right) \in \mathcal{C}(\mu, \mathcal{W})
$$

and assume that $\left(f_{\infty}, u_{\infty}\right) \in L^{2}(\mu) \oplus \mathscr{K}$ is the limit of the sequence $\left\{\left(f_{k}, u_{k}\right)\right\}_{k=0}^{\infty}$ :

$$
\left(f_{k}, u_{k}\right) \underset{k \rightarrow \infty}{\stackrel{\text { in } L^{2}(\mu) \oplus \mathscr{K}}{\longrightarrow}}\left(f_{\infty}, u_{\infty}\right) .
$$

We want to show that $\left(f_{\infty}, u_{\infty}\right) \in \mathcal{C}(\mu, \mathcal{W})$. Indeed, (2.28) implies, up to passing to a subsequence if necessary, that

$$
f_{k}(t)=\sum_{n=0}^{\infty} a_{n}^{(k)} t^{n} \stackrel{k \rightarrow \infty}{\longrightarrow} f_{\infty}(t) \quad \text { for } \mu \text {-a.e. } t \in \mathbb{R}_{+} .
$$

Note that the condition $\mu\left(\mathbb{R}_{+} \backslash\left[0, s_{\mu}\right)\right)=0$ implies in particular that the support $\operatorname{supp}(\mu)$ is an infinite subset of $\mathbb{R}_{+}$. By $(2.29)$, there exists a sequence $\tau_{0}<\tau_{1}<\cdots$ in $\left[0, s_{\mu}\right)$ such that

$$
\lim _{m \rightarrow \infty} \tau_{m}=s_{\mu}
$$

and

$$
f_{k}\left(\tau_{m}\right)=\sum_{n=0}^{\infty} a_{n}^{(k)} \tau_{m}^{n} \stackrel{k \rightarrow \infty}{\longrightarrow} f_{\infty}\left(\tau_{m}\right) \in[0, \infty) \text { for all } m \in \mathbb{N} .
$$

It follows that

$$
M_{m}:=\sup _{k} \sum_{n=0}^{\infty} a_{n}^{(k)} \tau_{m}^{n} \in[0, \infty) \quad \text { for all } m \in \mathbb{N} .
$$

Since all coefficients $a_{n}^{(k)} \geq 0$, we have

$$
0 \leq a_{n}^{(k)} \tau_{m}^{n} \leq M_{m} \quad \text { for all } m, k \in \mathbb{N} .
$$

Using the compactness of $\left[0, M_{m}\right]$ and the canonical Cantor's diagonal method, we may extract a subsequence of positive integers, denoted by $0<k_{1}<k_{2}<k_{3}<\cdots$, such that for all $m, n \in \mathbb{N}$, the following limits exist:

$$
\lim _{i \rightarrow \infty} a_{n}^{\left(k_{i}\right)} \tau_{m}^{n} \in\left[0, M_{m}\right] .
$$

But this means that the $\operatorname{limits}_{\lim _{i \rightarrow \infty}} a_{n}^{\left(k_{i}\right)}$ exist for all $n \in \mathbb{N}$ and moreover,

$$
a_{n}^{(\infty)}:=\lim _{i \rightarrow \infty} a_{n}^{\left(k_{i}\right)} \in\left[0, \frac{M_{m}}{\tau_{m}^{n}}\right] \text { for all } m, n \in \mathbb{N} .
$$


Now for any $t \in\left[0, \tau_{m-1}\right]$, we have

$$
0 \leq \frac{t}{\tau_{m}} \leq \frac{\tau_{m-1}}{\tau_{m}}<1
$$

and hence by (2.30),

$$
\sum_{n=0}^{\infty} \sup _{i}\left|a_{n}^{\left(k_{i}\right)} t^{n}\right| \leq \sum_{n=0}^{\infty} \frac{M_{m}}{\tau_{m}^{n}} t^{n} \leq \sum_{n=0}^{\infty} M_{m}\left(\frac{\tau_{m-1}}{\tau_{m}}\right)^{n}<\infty \quad \text { for all } t \in\left[0, \tau_{m-1}\right] .
$$

Therefore, by the Dominated Convergence Theorem,

$$
\lim _{i \rightarrow \infty} \sum_{n=0}^{\infty} a_{n}^{\left(k_{i}\right)} t^{n}=\sum_{n=0}^{\infty} \lim _{i \rightarrow \infty} a_{n}^{\left(k_{i}\right)} t^{n}=\sum_{n=0}^{\infty} a_{n}^{(\infty)} t^{n} \quad \text { for all } t \in\left[0, \tau_{m-1}\right] .
$$

Combining (2.27), (2.29) and (2.32), we obtain

$$
f_{\infty}(t)=\sum_{n=0}^{\infty} a_{n}^{(\infty)} t^{n} \quad \text { for } \mu_{m-1} \text {-a.e. } t \in\left[0, \tau_{m-1}\right]
$$

where $\mu_{m-1}=\left.\mu\right|_{\left[0, \tau_{m-1}\right]}$ is the restiction of the measure $\mu$ on $\left[0, \tau_{m-1}\right]$. Since $m$ is arbitrary, we have

$$
f_{\infty}(t)=\sum_{n=0}^{\infty} a_{n}^{(\infty)} t^{n} \quad \text { for } \mu \text {-a.e. } t \in\left[0, s_{\mu}\right) .
$$

Combining (2.33) with the condition $\mu\left(\mathbb{R}_{+} \backslash\left[0, s_{\mu}\right)\right)=0$, we obtain

$$
f_{\infty}(t)=\sum_{n=0}^{\infty} a_{n}^{(\infty)} t^{n} \quad \text { for } \mu \text {-a.e. } t \in \mathbb{R}_{+} .
$$

We then need to show that the $\mu$-almost everywhere equality (2.34) implies the following $L^{2}$-norm convergence:

$$
\lim _{N \rightarrow \infty}\left\|\sum_{n=0}^{N} a_{n}^{(\infty)} t^{n}-f_{\infty}\right\|_{L^{2}(\mu)}=0
$$

But this again follows from the Dominated Convergence Theorem. Indeed, since $a_{n}^{(\infty)} \geq 0$ we have

$$
\sup _{N}\left(\sum_{n=N+1}^{\infty} a_{n}^{(\infty)} t^{n}\right)^{2} \leq\left(\sum_{n=0}^{\infty} a_{n}^{(\infty)} t^{n}\right)^{2}=f_{\infty}(t)^{2} .
$$

The above inequality combined with the assumption $f_{\infty} \in L^{2}\left(\mathbb{R}_{+}, \mu\right)$ implies

$$
\lim _{N \rightarrow \infty}\left\|\sum_{n=0}^{N} a_{n}^{(\infty)} t^{n}-f_{\infty}\right\|_{L^{2}(\mu)}^{2}=\lim _{N \rightarrow \infty} \int_{\mathbb{R}_{+}}\left(\sum_{n=N+1}^{\infty} a_{n}^{(\infty)} t^{n}\right)^{2} d \mu(t)=0 .
$$

Finally, it remains to show that

$$
\lim _{N \rightarrow \infty}\left\|\sum_{n=0}^{N} a_{n}^{(\infty)} w_{n}-u_{\infty}\right\|=0 .
$$


By the assumption (1.2) and the fact that $a_{n}^{(\infty)} \geq 0$ for all $n \in \mathbb{N}$, we have

$$
\left\|\sum_{n=N}^{M} a_{n}^{(\infty)} w_{n}\right\| \leq C\left\|\sum_{n=N}^{M} a_{n}^{(\infty)} t^{n}\right\|_{L^{2}(\mu)} \text { for all } N, M \in \mathbb{N} \text { with } N \leq M .
$$

Thus the convergence of the series $\sum_{n=0}^{\infty} a_{n}^{(\infty)} t^{n}$ in the space $L^{2}(\mu)$ implies the convergence of the series $\sum_{n=0}^{\infty} a_{n}^{(\infty)} w_{n}$ in $\mathscr{K}$. By (2.28), we have

$$
u_{\infty}=\lim _{k \rightarrow \infty} \sum_{n=0}^{\infty} a_{n}^{(k)} w_{n}
$$

Let $\left\{k_{i}\right\}_{i}$ be the subsequence of positive integers chosen as above. For any $N \in \mathbb{N}$ and any $i \in \mathbb{N}$, by the assumption (1.2) and the fact that $a_{n}^{\left(k_{i}\right)} \geq 0$, we have

$$
\begin{aligned}
\left\|\sum_{n=0}^{N} a_{n}^{(\infty)} w_{n}-u_{\infty}\right\| \leq & \left\|\sum_{n=0}^{N} a_{n}^{(\infty)} w_{n}-\sum_{n=0}^{N} a_{n}^{\left(k_{i}\right)} w_{n}\right\|+\left\|\sum_{n=N+1}^{\infty} a_{n}^{\left(k_{i}\right)} w_{n}\right\| \\
& +\left\|\sum_{n=0}^{\infty} a_{n}^{\left(k_{i}\right)} w_{n}-u_{\infty}\right\| \\
\leq & \sum_{n=0}^{N}\left|a_{n}^{(\infty)}-a_{n}^{\left(k_{i}\right)}\right| \cdot\left\|w_{n}\right\|+C\left\|\sum_{n=N+1}^{\infty} a_{n}^{\left(k_{i}\right)} t^{n}\right\|_{L^{2}(\mu)} \\
& +\left\|\sum_{n=0}^{\infty} a_{n}^{\left(k_{i}\right)} w_{n}-u_{\infty}\right\| .
\end{aligned}
$$

Combining (2.31), (2.37) and (2.38), for any $N \in \mathbb{N}$, we have

$$
\left\|\sum_{n=0}^{N} a_{n}^{(\infty)} w_{n}-u_{\infty}\right\| \leq C \liminf _{i \rightarrow \infty}\left\|\sum_{n=N+1}^{\infty} a_{n}^{\left(k_{i}\right)} t^{n}\right\|_{L^{2}(\mu)} .
$$

Note also that (2.28) and (2.35) together imply

$$
\lim _{i \rightarrow \infty}\left\|\sum_{n=0}^{\infty} a_{n}^{\left(k_{i}\right)} t^{n}-\sum_{n=0}^{\infty} a_{n}^{(\infty)} t^{n}\right\|_{L^{2}(\mu)}=0 .
$$

Therefore, for any fixed $N \in \mathbb{N}$, we have

$$
\liminf _{i \rightarrow \infty}\left\|\sum_{n=N+1}^{\infty} a_{n}^{\left(k_{i}\right)} t^{n}\right\|_{L^{2}(\mu)}=\left\|\sum_{n=N+1}^{\infty} a_{n}^{(\infty)} t^{n}\right\|_{L^{2}(\mu)}
$$

and hence

$$
\left\|\sum_{n=0}^{N} a_{n}^{(\infty)} w_{n}-u_{\infty}\right\| \leq C\left\|\sum_{n=N+1}^{\infty} a_{n}^{(\infty)} t^{n}\right\|_{L^{2}(\mu)}
$$

Thus we have

$$
\limsup _{N \rightarrow \infty}\left\|\sum_{n=0}^{N} a_{n}^{(\infty)} w_{n}-u_{\infty}\right\| \leq C \limsup _{N \rightarrow \infty}\left\|\sum_{n=N+1}^{\infty} a_{n}^{(\infty)} t^{n}\right\|_{L^{2}(\mu)}=0 .
$$

This completes the proof of the limit relation (2.36). 
Remark. The implication $(2.25) \Longrightarrow(2.27)$ relies heavily on the non-negativity of the functions $a_{n} t^{n}$ on $\left[0, s_{\mu}\right)$. In general, the equality $f=\sum_{n=0}^{\infty} f_{n}$ in the Hilbert space $L^{2}(\mu)$ does not imply the $\mu$-almost everywhere equality $f(t) \stackrel{\mu \text {-a.e. }}{=} \sum_{n=0}^{\infty} f_{n}(t)$.

Proof of Corollary 1.2. By writing $\mu=\left.\nu\right|_{\mathbb{R}_{+}}$, we have

$$
L^{2}(\mathbb{R}, \nu)=L^{2}\left(\mathbb{R}_{+}, \mu\right) \oplus L^{2}\left(\mathbb{R}_{-}, \nu\right) .
$$

The assumption (1.4) implies that for any finitely supported sequence $\left\{a_{n}\right\}_{n=0}^{\infty}$ of nonnegative numbers, we have

$$
\begin{aligned}
\left\|\sum_{n=0}^{\infty} a_{n} t^{n}\right\|_{L^{2}\left(\mathbb{R}_{-}, \nu\right)}^{2} & =\sum_{n, m \geq 0} a_{n} a_{m} \int_{\mathbb{R}_{-}} t^{m+n} d \nu(t) \\
& \leq \sum_{\substack{m, n \geq 0 \\
m+n \text { is even }}} a_{n} a_{m} \int_{\mathbb{R}_{+}} t^{m+n} d \mu(t) \\
& \leq C \sum_{m, n \geq 0} a_{n} a_{m} \int_{\mathbb{R}_{+}} t^{m+n} d \mu(t)=C\left\|\sum_{n=0}^{\infty} a_{n} t^{n}\right\|_{L^{2}\left(\mathbb{R}_{+}, \mu\right)}^{2}
\end{aligned}
$$

where in the first inequality, we have used the inequalities

$$
\int_{\mathbb{R}_{-}} t^{2 k+1} d \nu(t) \leq 0 \quad \text { for all } k \in \mathbb{N} .
$$

Now Corollary 1.2 follows immediately from Theorem 1.1.

\section{Characterization of the metric projection}

3.1. Proof of Proposition 1.3. Recall that $\mathcal{C}[[\mathcal{V}]]$ is assumed to be closed. For any $w \in \mathscr{H}$, by a classical result on the metric projections onto a closed convex set (cf. [16, Lemma 1.1]), $P_{\mathcal{C}[[\mathcal{V}]]}(w)$ is uniquely determined by

$$
\left\{\begin{array}{l}
P_{\mathcal{C}[\mathcal{V}]]}(w) \in \mathcal{C}[[\mathcal{V}]], \\
\left\langle w-P_{\mathcal{C}[[\mathcal{V}]]}(w), u-P_{\mathcal{C}[[\mathcal{V}]]}(w)\right\rangle \leq 0, \quad \text { for all } u \in \mathcal{C}[[\mathcal{V}]] .
\end{array}\right.
$$

By the assumption that $\mathcal{C}[[\mathcal{V}]]$ is a closed convex cone, we may use [9, Lemma 3] to obtain

$$
\left\langle w-P_{\mathcal{C}[[\mathcal{V}]]}(w), P_{\mathcal{C}[[\mathcal{V}]]}(w)\right\rangle=0 .
$$

This combined with (3.39) implies that $P_{\mathcal{C}[\mathcal{V}]]}(w)$ is uniquely determined by

$$
\left\{\begin{array}{l}
P_{\mathcal{C}[[\mathcal{V}]]}(w) \in \mathcal{C}[[\mathcal{V}]], \\
\left\langle w-P_{\mathcal{C}[[\mathcal{V}]]}(w), P_{\mathcal{C}[[\mathcal{V}]]}(w)\right\rangle=0, \\
\left\langle w-P_{\mathcal{C}[[\mathcal{V}]]}(w), u\right\rangle \leq 0, \quad \text { for all } u \in \mathcal{C}[[\mathcal{V}]] .
\end{array}\right.
$$

By the definition of $\mathcal{C}[[\mathcal{V}]]$, the condition

$$
\left\langle w-P_{\mathcal{C}[[\mathcal{V}]]}(w), u\right\rangle \leq 0 \quad \text { for any } u \in \mathcal{C}[[\mathcal{V}]]
$$

is satisfied if and only if

$$
\left\langle w-P_{\mathcal{C}[\mathcal{V}]]}(w), v_{n}\right\rangle \leq 0 \quad \text { for all } n \in \mathbb{N} .
$$


By writing

$$
P_{\mathcal{C}[\mathcal{V}]]}(w)=\sum_{n=0}^{\infty} a_{n} v_{n} \quad \text { with } a_{n} \geq 0 \text { for all } n \in \mathbb{N}
$$

we have

$$
0=\left\langle w-P_{\mathcal{C}[[\mathcal{V}]]}(w), P_{\mathcal{C}[[\mathcal{V}]]}(w)\right\rangle=\sum_{n=0}^{\infty} a_{n}\left\langle w-P_{\mathcal{C}[[\mathcal{V}]]}(w), v_{n}\right\rangle .
$$

Combining (3.41) and (3.42), we obtain

$$
\left\langle w-P_{\mathcal{C}[[\mathcal{V}]]}(w), v_{n}\right\rangle=0 \quad \text { for all those } n \in \mathbb{N} \text { such that } a_{n}>0 .
$$

On the other hand, (3.43) clearly implies the equality (3.42). Therefore, the condition (3.40) is equivalent to

$$
\begin{cases}P_{\mathcal{C}[[\mathcal{V}]]}(w)=\sum_{n=0}^{\infty} a_{n} v_{n} \in \mathcal{C}[[\mathcal{V}]], & \text { for all } n \in \mathbb{N} \text { such that } a_{n}>0, \\ \sum_{k \in \mathbb{N}} a_{k}\left\langle v_{k}, v_{n}\right\rangle=\left\langle w, v_{n}\right\rangle, & \text { for all } n \in \mathbb{N} . \\ \sum_{k \in \mathbb{N}} a_{k}\left\langle v_{k}, v_{n}\right\rangle \geq\left\langle w, v_{n}\right\rangle, & \end{cases}
$$

This completes the proof of Proposition 1.3.

3.2. Proof of Corollary 1.5. If $\mathcal{V} \subset \mathscr{H}$ is a finite set, then the convex cone $\mathcal{C}[\mathcal{V}]$ generated by $\mathcal{V}$ is always closed, cf. e.g. [16, p. 236] and [3, p. 25].

Lemma 3.1. Let $X_{1}, \cdots, X_{n}$ be linear independent real-valued random variables, all of which are of finite second moment. Then for any $c=\left(c_{1}, \cdots, c_{n}\right) \in \mathbb{R}^{n}$, there exists a real-valued random variable $Y$ of finite second moment such that

$$
c_{i}=\mathbb{E}\left[Y X_{i}\right], \quad 1 \leq i \leq n .
$$

Proof. Set

$$
A=\left(\mathbb{E}\left(X_{i} X_{j}\right)\right)_{1 \leq i, j \leq n}=\mathbb{E}\left[\left(\begin{array}{c}
X_{1} \\
\vdots \\
X_{n}
\end{array}\right)\left(X_{1} \cdots X_{n}\right)\right] .
$$

Since $X_{1}, \cdots, X_{n}$ are linear independent, by Schmidt orthogonalization method, there exists a non-singular matrix $P$ such that the random variables $Z_{i}$ 's defined by

$$
\left(\begin{array}{c}
Z_{1} \\
\vdots \\
Z_{n}
\end{array}\right)=P\left(\begin{array}{c}
X_{1} \\
\vdots \\
X_{n}
\end{array}\right)
$$

are orthogonal and $\mathbb{E}\left(Z_{i}^{2}\right)>0$ for all $i \in\{1, \cdots, n\}$. Note that the condition (3.44) can be written as

$$
\left(\begin{array}{c}
c_{1} \\
\vdots \\
c_{n}
\end{array}\right)=\mathbb{E}\left[\left(\begin{array}{c}
X_{1} \\
\vdots \\
X_{n}
\end{array}\right) Y\right]
$$


Since $P$ is non-singular, $(3.45)$ is equivalent to

$$
\left(\begin{array}{c}
c_{1}^{\prime} \\
\vdots \\
c_{n}^{\prime}
\end{array}\right):=P\left(\begin{array}{c}
c_{1} \\
\vdots \\
c_{n}
\end{array}\right)=P \mathbb{E}\left[\left(\begin{array}{c}
X_{1} \\
\vdots \\
X_{n}
\end{array}\right) Y\right]=\mathbb{E}\left[\left(\begin{array}{c}
Z_{1} \\
\vdots \\
Z_{n}
\end{array}\right) Y\right]
$$

If we set

$$
Y=\sum_{i=1}^{n} \frac{c_{i}^{\prime} Z_{i}}{\mathbb{E}\left(Z_{i}^{2}\right)}
$$

then $Y$ satisfies the condition (3.46). This completes the whole proof.

Proof of Corollary 1.5. Since $A$ is a non-singular positive definite matrix, there exist realvalued square-integrable and linear independent random variables $X_{1}, \cdots, X_{n}$ such that

$$
\mathbb{E}\left(X_{i}\right)=0 \text { and } A=\left(\mathbb{E}\left(X_{i} X_{j}\right)\right)_{1 \leq i, j \leq n}
$$

By Lemma 3.1, there exists a real-valued square-integrable random variable $Y$ such that

$$
c_{i}=\mathbb{E}\left(X_{i} Y\right), \quad 1 \leq i \leq n .
$$

The convex cone in the associated Hilbert space of square-integrable random variables generated by $X_{1}, \cdots, X_{n}$ is

$$
\mathcal{C}\left(X_{1}, \cdots, X_{n}\right)=\left\{\sum_{i=1}^{n} b_{i} X_{i} \mid b_{i} \geq 0\right\} .
$$

Since $\mathcal{C}\left(X_{1}, \cdots, X_{n}\right)$ is closed (cf. [16, p. 236] and [3, p. 25]), there exists a unique $Z \in \mathcal{C}\left(X_{1}, \cdots, X_{n}\right)$ closest to $Y$. Write

$$
Z=\sum_{i=1}^{n} b_{i} X_{i}=\sum_{i \in S} b_{i} X_{i} \quad \text { with } b_{i}>0 \text { for all } i \in S .
$$

By Proposition 1.3, $S$ and the coefficients $\left(b_{i}\right)_{i \in S}$ are uniquely determined by

$$
\begin{cases}b_{i}>0, & \text { for all } i \in S \\ \left\langle\sum_{i \in S} b_{i} X_{i}, X_{j}\right\rangle=\left\langle Y, X_{j}\right\rangle=c_{j}, & \text { for all } j \in S \\ \left\langle\sum_{i \in S} b_{i} X_{i}, X_{j}\right\rangle \geq\left\langle Y, X_{j}\right\rangle=c_{j}, & \text { for all } j \in\{1,2, \cdots, n\} .\end{cases}
$$

In other words, $S$ and $\left(b_{j}\right)_{j \in S}$ are uniquely determined by

$$
\begin{cases}b_{i}>0, & \text { for all } i \in S, \\ \sum_{i \in S} a_{i j} b_{i}=c_{j}, & \text { for all } j \in S, \\ \sum_{i \in S} a_{i j} b_{i} \geq c_{j}, & \text { for all } j \in\{1,2, \cdots, n\} .\end{cases}
$$

By noting $a_{i j}=a_{j i}$, we complete the whole proof. 


\section{Applications in function theory}

\subsection{Power functions and signed power functions.}

Proof of Proposition 1.6. Let $\alpha \in[0,2), m \in \mathbb{N}$ and $\left(a_{m}, b_{m}\right)$ be defined as (1.12). By Proposition 1.3, for proving the equality (1.11), it suffices to verify

$$
\left\langle a_{m} t^{2 m}+b_{m} t^{2 m+2}, t^{j}\right\rangle_{L^{2}([-1,1])} \geq\left\langle|t|^{2 m+\alpha}, t^{j}\right\rangle_{L^{2}([-1,1])} \quad \text { for all } j \in \mathbb{N}
$$

and

$$
\left\langle a_{m} t^{2 m}+b_{m} t^{2 m+2}, t^{j}\right\rangle_{L^{2}([-1,1])}=\left\langle|t|^{2 m+\alpha}, t^{j}\right\rangle_{L^{2}([-1,1])} \quad \text { for } j \in\{2 m, 2 m+2\}
$$

If $j$ is an odd number, then (4.47) holds since both sides of (4.47) vanish; the same is true for (4.48). So we now focus on even numbers $j=2 k$ with $k \geq 0$. Note that $\left(a_{m}, b_{m}\right)$ defined by (1.12) is in fact the solution of the linear equation

$$
\left(\begin{array}{cc}
\frac{2}{4 m+1} & \frac{2}{4 m+3} \\
\frac{2}{4 m+3} & \frac{2}{4 m+5}
\end{array}\right)\left(\begin{array}{l}
a_{m} \\
b_{m}
\end{array}\right)=\left(\begin{array}{c}
\frac{2}{4 m+1+\alpha} \\
\frac{2}{4 m+3+\alpha}
\end{array}\right) .
$$

Since for any even number $j=2 k$, we have

$$
\left\langle|t|^{\beta}, t^{2 k}\right\rangle_{L^{2}([-1,1])}=\frac{2}{\beta+2 k+1} \quad \text { for all } \beta \geq 0
$$

and

$$
\left\langle t^{2 \ell}, t^{2 k}\right\rangle_{L^{2}([-1,1])}=\frac{2}{2 \ell+2 k+1} \text { for all } k, \ell \in \mathbb{N},
$$

the equality (4.49) is equivalent to the equality (4.48). It remains to show the inequalities (4.47) for all even numbers $j=2 k$ with $k \geq 0$. That is, we need to show

$$
\frac{2 a_{m}}{2 m+2 k+1}+\frac{2 b_{m}}{2 m+2 k+3} \geq \frac{2}{2 m+2 k+\alpha+1} \quad \text { for all } k \in \mathbb{N} \text {. }
$$

Set

$$
\begin{aligned}
D_{k}:=(4 m+1+\alpha)(4 m+ & 3+\alpha)(2 m+2 k+1)(2 m+2 k+3)(2 m+2 k+\alpha+1) \\
& \times\left(\frac{2 a_{m}}{2 m+2 k+1}+\frac{2 b_{m}}{2 m+2 k+3}-\frac{2}{2 m+2 k+\alpha+1}\right) .
\end{aligned}
$$

Then we only need to show that

$$
D_{k} \geq 0 \quad \text { for all } k \in \mathbb{N} \text {. }
$$

Write

$$
\tau=4 m+3 \text { and } x=4 k,
$$


then

$$
\begin{aligned}
D_{k}= & (2-\alpha)(4 m+1)(4 m+3)(2 m+2 k+3)(2 m+2 k+\alpha+1) \\
& +\alpha(4 m+3)(4 m+5)(2 m+2 k+1)(2 m+2 k+\alpha+1) \\
& -2(4 m+1+\alpha)(4 m+3+\alpha)(2 m+2 k+1)(2 m+2 k+\alpha+1) \\
= & (2-\alpha)(\tau-2) \tau\left(\frac{\tau+3}{2}+\frac{x}{2}\right)\left(\frac{\tau-3}{2}+\frac{x}{2}+\alpha+1\right) \\
& +\alpha \tau(\tau+2)\left(\frac{\tau-3}{2}+\frac{x}{2}+1\right)\left(\frac{\tau-3}{2}+\frac{x}{2}+\alpha+1\right) \\
& -2(\tau-2+\alpha)(\tau+\alpha)\left(\frac{\tau-3}{2}+\frac{x}{2}+1\right)\left(\frac{\tau-3}{2}+\frac{x}{2}+3\right) \\
= & \frac{1}{4}\left(A x^{2}+B x+C\right),
\end{aligned}
$$

where

$$
A=2 \alpha(2-\alpha), B=4 \alpha(\alpha-2)(\tau-1) \text { and } C=2 \alpha(2-\alpha)\left(\tau^{2}-2 \tau-3\right) \text {. }
$$

Therefore,

$$
D_{k}=\frac{1}{2} \alpha(2-\alpha)\left[(x-(\tau-1))^{2}-4\right]
$$

By substituting (4.51) into the above equality, we have

$$
D_{k}=2 \alpha(2-\alpha)\left[(2 k-2 m-1)^{2}-1\right] .
$$

By observing

$$
(2 k-2 m-1)^{2}-1 \geq 0 \text { for any } k, m \in \mathbb{N}
$$

and using the assumption $\alpha \in[0,2)$, we obtain the desired inequalities (4.50). This completes the proof of the equality (1.11).

Now we proceed to the proof of the equality (1.13). We have

$$
\begin{aligned}
{\left[d\left(h_{2 m+\alpha}, \mathcal{A}_{+}\right)\right]^{2}=} & \int_{[-1,1]}\left(|t|^{2 m+\alpha}-a_{m} t^{2 m}-b_{m} t^{2 m+2}\right)^{2} d t \\
= & 2 \int_{[0,1]}\left(t^{2 m+\alpha}-a_{m} t^{2 m}-b_{m} t^{2 m+2}\right)^{2} d t \\
= & \frac{2}{4 m+2 \alpha+1}+\frac{2 a_{m}^{2}}{4 m+1}+\frac{2 b_{m}^{2}}{4 m+5} \\
& +\frac{4 a_{m} b_{m}}{4 m+3}-\frac{4 a_{m}}{4 m+\alpha+1}-\frac{4 b_{m}}{4 m+\alpha+3} .
\end{aligned}
$$


By substituting $a_{m}, b_{m}$ defined in (1.12) and by writing again $\tau=4 m+3$, we obtain

$$
\begin{aligned}
{\left[d\left(h_{2 m+\alpha}, \mathcal{A}_{+}\right)\right]^{2}=} & \frac{2}{\tau+2 \alpha-2}+\frac{2}{\tau-2}\left(\frac{(\tau-2) \tau}{(\tau+\alpha-2)(\tau+\alpha)} \frac{2-\alpha}{2}\right)^{2} \\
& +\frac{2}{\tau+2}\left(\frac{\tau(\tau+2)}{(\tau+\alpha-2)(\tau+\alpha)} \frac{\alpha}{2}\right)^{2} \\
& +\frac{4}{\tau}\left(\frac{(\tau-2) \tau}{(\tau+\alpha-2)(\tau+\alpha)} \frac{2-\alpha}{2}\right)\left(\frac{\tau(\tau+2)}{(\tau+\alpha-2)(\tau+\alpha)} \frac{\alpha}{2}\right) \\
& -\frac{4}{\tau+\alpha-2}\left(\frac{(\tau-2) \tau}{(\tau+\alpha-2)(\tau+\alpha)} \frac{2-\alpha}{2}\right)-\frac{4}{\tau+\alpha}\left(\frac{\tau(\tau+2)}{(\tau+\alpha-2)(t+\alpha)} \frac{\alpha}{2}\right) \\
= & \frac{2}{\tau+2 \alpha-2}+\frac{2 \tau}{(\tau+\alpha-2)^{2}(\tau+\alpha)^{2}} H(\alpha, \tau),
\end{aligned}
$$

where

$$
\begin{aligned}
H(\alpha, \tau)= & \frac{(2-\alpha)^{2}}{4} \tau(\tau-2)+\frac{\alpha^{2}}{4} \tau(\tau+2)+\frac{\alpha(2-\alpha)}{2}(\tau-2)(\tau+2) \\
& -(2-\alpha)(\tau-2)(\tau+\alpha)-\alpha(\tau+2)(\tau+\alpha-2) \\
= & -\tau^{2}+(2-2 \alpha) \tau+2 \alpha(2-\alpha)=-\tau(\tau+2 \alpha-2)+2 \alpha(2-\alpha) .
\end{aligned}
$$

Therefore, we have

$$
\left[d\left(h_{2 m+\alpha}, \mathcal{A}_{+}\right)\right]^{2}=\frac{2}{(\tau+2 \alpha-2)(\tau+\alpha-2)^{2}(\tau+\alpha)^{2}} K(\alpha, \tau)
$$

with $K(\alpha, \tau)$ given by

$$
\begin{aligned}
K(\alpha, \tau) & =(\tau+\alpha-2)^{2}(\tau+\alpha)^{2}+\tau(\tau+2 \alpha-2) H(\alpha, \tau) \\
& =[\tau(\tau+2 \alpha-2)-\alpha(2-\alpha)]^{2}-\tau^{2}(\tau+2 \alpha-2)^{2}+2 \alpha(2-\alpha) \tau(\tau+2 \alpha-2) \\
& =\alpha^{2}(2-\alpha)^{2} .
\end{aligned}
$$

Thus we obtain

$$
\begin{aligned}
{\left[d\left(h_{2 m+\alpha}, \mathcal{A}_{+}\right)\right]^{2} } & =\frac{2 \alpha^{2}(2-\alpha)^{2}}{(\tau+2 \alpha-2)(\tau+\alpha-2)^{2}(\tau+\alpha)^{2}} \\
& =\frac{2 \alpha^{2}(2-\alpha)^{2}}{(4 m+2 \alpha+1)(4 m+\alpha+1)^{2}(4 m+\alpha+3)^{2}} .
\end{aligned}
$$

This completes the proof of the equality (1.13).

Proof of Corollary 1.7. Fix $m \in \mathbb{N}$ and a positive Radon measure $\nu$ on $[0,2]$. Set

$$
g_{m, \nu}:=\int_{[0,2]} h_{2 m+\alpha} d \nu(\alpha)
$$

and

$$
A_{m, \nu}:=\int_{[0,2]} a_{m}(\alpha) d \nu(\alpha) \geq 0, B_{m, \nu}:=\int_{[0,2]} b_{m}(\alpha) d \nu(\alpha) \geq 0 .
$$

By integrating the inequalities (4.47) and the equalities (4.48) against the measure $\nu$, we obtain

$$
\left\langle A_{m, \nu} t^{2 m}+B_{m, \nu} t^{2 m+2}, t^{j}\right\rangle_{L^{2}([-1,1])} \geq\left\langle g_{m, \nu}, t^{j}\right\rangle_{L^{2}([-1,1])} \quad \text { for all } j \in \mathbb{N}
$$


and

$$
\left\langle A_{m, \nu} t^{2 m}+B_{m, \nu} t^{2 m+2}, t^{j}\right\rangle_{L^{2}([-1,1])}=\left\langle g_{m, \nu}, t^{j}\right\rangle_{L^{2}([-1,1])} \quad \text { for } j \in\{2 m, 2 m+2\} .
$$

Thus by Proposition 1.3, we obtain the desired equality

$$
P_{\mathcal{A}_{+}}\left(g_{m, \nu}\right)=A_{m, \nu} t^{2 m}+B_{m, \nu} t^{2 m+2} .
$$

Proof of Proposition 1.8. Let $\alpha \in[0,2), m \in \mathbb{N}$ and $\left(c_{m}, d_{m}\right)$ be defined as (1.17). By Proposition 1.3, for proving the equality (1.16), it suffices to verify

$$
\left\langle c_{m} t^{2 m+1}+d_{m} t^{2 m+3}, t^{j}\right\rangle_{L^{2}([-1,1])} \geq\left\langle\operatorname{sgn}(t)|t|^{2 m+1+\alpha}, t^{j}\right\rangle_{L^{2}([-1,1])} \quad \text { for all } j \in \mathbb{N}
$$

and

$\left\langle c_{m} t^{2 m+1}+d_{m} t^{2 m+3}, t^{j}\right\rangle_{L^{2}([-1,1])}=\left\langle\operatorname{sgn}(t)|t|^{2 m+1+\alpha}, t^{j}\right\rangle_{L^{2}([-1,1])}$ for $j \in\{2 m+1,2 m+3\}$.

If $j$ is an even number, then (4.52) holds since both sides of (4.52) vanish; the same is true for (4.53). So we now focus on odd numbers $j=2 k+1$ with $k \geq 0$. Note that $\left(c_{m}, d_{m}\right)$ defined by $(1.17)$ is in fact the solution of the linear equation

$$
\left(\begin{array}{cc}
\frac{2}{4 m+3} & \frac{2}{4 m+5} \\
\frac{2}{4 m+5} & \frac{2}{4 m+7}
\end{array}\right)\left(\begin{array}{c}
c_{m} \\
d_{m}
\end{array}\right)=\left(\begin{array}{c}
\frac{2}{4 m+\alpha+3} \\
\frac{2}{4 m+\alpha+5}
\end{array}\right) .
$$

Since for any odd number $j=2 k+1$,

$$
\left\langle\operatorname{sgn}(t)|t|^{\gamma}, t^{2 k+1}\right\rangle_{L^{2}([-1,1])}=\frac{2}{\gamma+2 k+2} \quad \text { for all } \gamma \geq 0
$$

and

$$
\left\langle t^{2 \ell+1}, t^{2 k+1}\right\rangle_{L^{2}([-1,1])}=\frac{2}{2 \ell+2 k+3} \text { for all } k, \ell \in \mathbb{N},
$$

the equality (4.54) is equivalent to the equality (4.53). It remains to show the inequalities (4.52) for all odd numbers $j=2 k+1$ with $k \geq 0$. That is, we need to show

$$
\frac{2 c_{m}}{2 m+2 k+3}+\frac{2 d_{m}}{2 m+2 k+5} \geq \frac{2}{2 m+2 k+\alpha+3} \quad \text { for all } k \in \mathbb{N} .
$$

Set

$$
\begin{aligned}
T_{k}:=(2 m+2 k+3)(2 m & +2 k+5)(4 m+3+\alpha)(4 m+5+\alpha)(2 m+2 k+3+\alpha) \\
& \times\left(\frac{2 c_{m}}{2 m+2 k+3}+\frac{2 d_{m}}{2 m+2 k+5}-\frac{2}{2 m+2 k+3+\alpha}\right) .
\end{aligned}
$$

Then we only need to show

$$
T_{k} \geq 0 \quad \text { for all } k \in \mathbb{N} \text {. }
$$

By using exactly the same arguments as those in dealing with $D_{k}$ in the proof of Proposition 1.6 (another simpler way is to replace everywhere the pair $(m, k)$ in the definition of $D_{k}$ by the pair $\left(m+\frac{1}{2}, k+\frac{1}{2}\right)$ to obtain a reduced form of $T_{k}$ ), we obtain

$$
T_{k}=2 \alpha(2-\alpha)\left[(2 k-2 m-1)^{2}-1\right] \quad \text { for all } k \in \mathbb{N} \text {. }
$$


By observing $(2 k-2 m-1)^{2}-1 \geq 0$ for any $k, m \in \mathbb{N}$ and using the assumption $\alpha \in[0,2)$, we obtain the desired inequalities (4.55). This completes the proof of the equality (1.16).

The verification of the equality (1.18) is similar to that of the equality (1.13).

4.2. Indicator functions. For any $n \in \mathbb{N}$, define an $(n+1) \times(n+1)$-matrix by

$$
M_{n}:=\left(\left\langle t^{i}, t^{j}\right\rangle_{L^{2}([-1,1])}\right)_{0 \leq i, j \leq n}
$$

By the linear independence of the functions $1, t, t^{2}, \cdots$ on $[-1,1]$, for any $n \in \mathbb{N}$, the matrix $M_{n}$ is non-singular.

Note that for any $n \in \mathbb{N}$ and any $a \in[-1,1)$, we have

$$
\left\langle\psi_{a}, t^{n}\right\rangle_{L^{2}([-1,1])}=\int_{a}^{1} t^{n} d t=\frac{1-a^{n+1}}{n+1} .
$$

Let $v_{a}^{(n)} \in \mathbb{R}^{n+1}$ be the column vector defined by

$$
\begin{aligned}
v_{a}^{(n)} & =\left(\left\langle\psi_{a}, 1\right\rangle_{L^{2}([-1,1])},\left\langle\psi_{a}, t\right\rangle_{L^{2}([-1,1])}, \cdots,\left\langle\psi_{a}, t^{n}\right\rangle_{L^{2}([-1,1])}\right)^{\top} \\
& =\left(1-a, \frac{1-a^{2}}{2}, \cdots, \frac{1-a^{n+1}}{n+1}\right)^{\top} .
\end{aligned}
$$

Denote by $\mathbb{R}_{+}^{*}=(0, \infty)$ the set of all positive numbers. Lemmas 4.1 and 4.2 below will be used in the proof of Propositions 1.9 and 1.10 .

Lemma 4.1. Assume that $a \in[-1,1)$. Then the linear equation

$$
M_{2} x=v_{a}^{(2)}
$$

has a solution in $\left(\mathbb{R}_{+}^{*}\right)^{3}$ if and only if

$$
0<a<\frac{\sqrt{105}-5}{10}
$$

Proof. The solution $x=\left(x_{0}, x_{1}, x_{2}\right)$ of the linear equation (4.56) is given by

$$
\left\{\begin{array}{l}
x_{0}=\frac{1}{8}\left(4-9 a+5 a^{3}\right)=\frac{1}{8}(1-a)\left(4-5 a-5 a^{2}\right) \\
x_{1}=\frac{3}{4}\left(1-a^{2}\right) \\
x_{2}=\frac{15}{8}\left(a-a^{3}\right)=\frac{15}{8} a\left(1-a^{2}\right)
\end{array} .\right.
$$

A simple computation shows that, under the assumption $a \in[-1,1)$, the solution $x$ belongs to $\left(\mathbb{R}_{+}^{*}\right)^{3}$ if and only if $0<a<\frac{\sqrt{105}-5}{10}$. This completes the proof of the lemma.

Lemma 4.2. Assume that $\rho \in[0,1)$. Then the condition

$$
\left(1-3 \rho+2 \rho^{n+1}\right) n+3 \rho^{n+1} \geq 3 \rho \quad \text { for all integers } n \geq 1
$$

holds if and only if $\rho \in\left[0, \frac{1}{5}\right]$.

Proof. Assume that (4.58) holds. Then by taking $n=1$, we have

$$
1-3 \rho+2 \rho^{2}+3 \rho^{2} \geq 3 \rho .
$$

This combined with the assumption $\rho \in[0,1)$ implies that $\rho \in[0,1 / 5]$.

Conversely, assume that $\rho \in[0,1 / 5]$. Then for $n=1$, we have

$$
1-3 \rho+2 \rho^{2}+3 \rho^{2}-3 \rho=(1-\rho)(1-5 \rho) \geq 0 .
$$


This implies the inequality (4.58) for $n=1$. Now assume that $n \geq 2$, we have

$$
\left(1-3 \rho+2 \rho^{n+1}\right) n+3 \rho^{n+1}-3 \rho \geq\left(1-\frac{3}{5}\right) n-\frac{3}{5}=\frac{2 n-3}{5} \geq \frac{1}{5} \geq 0 .
$$

This implies the inequality (4.58) for all integers $n \geq 2$.

Proof of Proposition 1.9. Let $x_{0}, x_{1}, x_{2}$ be given as in (4.57) and recall that $x$ is the solution to the linear equation (4.56). By the discussions in $\S 1.3$ and Proposition 1.3, the equality (1.19) holds if and only if the following conditions are all satisfied:

- $x \in\left(\mathbb{R}_{+}^{*}\right)^{3}$;

- for all $j \in\{0,1,2\}$,

$$
\left\langle\sum_{i=0}^{2} x_{i} t^{i}, t^{j}\right\rangle_{L^{2}([-1,1])}=\left\langle\psi_{a}, t^{j}\right\rangle_{L^{2}([-1,1])}=\frac{1-a^{j+1}}{j+1}
$$

- for all integers $j \geq 3$,

$$
\left\langle\sum_{i=0}^{2} x_{i} t^{i}, t^{j}\right\rangle_{L^{2}([-1,1])} \geq\left\langle\psi_{a}, t^{j}\right\rangle_{L^{2}([-1,1])}=\frac{1-a^{j+1}}{j+1} .
$$

Note that the system of linear equations (4.59) for $j \in\{0,1,2\}$ is equivalent to the linear equation (4.56). Thus by the definition of $x$, the equalities (4.59) hold for all $j \in\{0,1,2\}$. By Lemma 4.1, $x \in\left(\mathbb{R}_{+}^{*}\right)^{3}$ if and only if $0<a<\frac{\sqrt{105}-5}{10}$. Now let us analyze (4.60). If $j=2 n+1$ with $n \geq 1$, then

$$
\begin{aligned}
& \left\langle\sum_{i=0}^{2} x_{i} t^{i}, t^{2 n+1}\right\rangle_{L^{2}([-1,1])}-\left\langle\psi_{a}, t^{2 n+1}\right\rangle_{L^{2}([-1,1])}=\frac{2}{2 n+3} \cdot \frac{3}{4}\left(1-a^{2}\right)-\frac{1-a^{2 n+2}}{2 n+2} \\
& =\frac{\left(1-3 a^{2}+2 a^{2 n+2}\right) n+3 a^{2 n+2}-3 a^{2}}{2(2 n+3)(n+1)}
\end{aligned}
$$

and if $j=2 n+2$ with $n \geq 1$, then

$$
\begin{aligned}
& \left\langle\sum_{i=0}^{2} x_{i} t^{i}, t^{2 n+2}\right\rangle_{L^{2}([-1,1])}-\left\langle\psi_{a}, t^{2 n+2}\right\rangle_{L^{2}([-1,1])} \\
= & \frac{2}{2 n+3} \cdot \frac{1}{8}\left(4-9 a+5 a^{3}\right)+\frac{2}{2 n+5} \cdot \frac{15}{8}\left(a-a^{3}\right)-\frac{1-a^{2 n+3}}{2 n+3} \\
= & \frac{a\left[\left(3-5 a^{2}+2 a^{2 n+2}\right) n-5 a^{2}+5 a^{2 n+2}\right]}{(2 n+3)(2 n+5)} .
\end{aligned}
$$

Therefore, the equality (1.19) holds if and only if the following conditions are all satisfied:

- $0<a<\frac{\sqrt{105}-5}{10}$;

- for all integers $n \geq 1$,

$$
\left(1-3 a^{2}+2 a^{2 n+2}\right) n+3 a^{2 n+2}-3 a^{2} \geq 0 ;
$$

- for all integers $n \geq 1$,

$$
a\left[\left(3-5 a^{2}+2 a^{2 n+2}\right) n-5 a^{2}+5 a^{2 n+2}\right] \geq 0 .
$$


Observe that

$$
\begin{aligned}
\left(3-5 a^{2}+2 a^{2 n+2}\right) n+5 a^{2 n+2}-5 a^{2}=3 & {\left[\left(1-3 a^{2}+2 a^{2 n+2}\right) n+3 a^{2 n+2}-3 a^{2}\right] } \\
& +4 a^{2}(n+1)\left(1-a^{2 n}\right) \\
\geq 3 & \left.\left(1-3 a^{2}+2 a^{2 n+2}\right) n+3 a^{2 n+2}-3 a^{2}\right] .
\end{aligned}
$$

Thus by Lemma 4.2 and the inequality $\frac{1}{\sqrt{5}}<\frac{\sqrt{105}-5}{10}$, the equality (1.19) holds if and only if $0<a \leq \frac{1}{\sqrt{5}}$.

Finally, by Proposition 1.3, the above arguments imply that for $0<a \leq \frac{1}{\sqrt{5}}$, we have

$$
P_{\mathcal{A}_{+}}\left(\psi_{a}\right)=\sum_{i=0}^{2} x_{i} t^{i}
$$

This is the desired equality (1.20).

Proof of Corollary 1.12. The proof of Corollary 1.12 is similar to that of Corollary 1.7.

Proof of Proposition 1.10. Set

$$
y_{0}:=\frac{1-a}{2}, \quad y_{1}:=\frac{3}{4}\left(1-a^{2}\right) .
$$

Then the assumption $a \in[-1,1)$ implies that $y=\left(y_{0}, y_{1}\right) \in\left(\mathbb{R}_{+}^{*}\right)^{2}$. Therefore, by Proposition 1.3, the equality (1.22) holds if and only if

$$
\left\langle\sum_{i=0}^{1} y_{i} t^{i}, t^{j}\right\rangle_{L^{2}([-1,1])}=\left\langle\psi_{a}, t^{j}\right\rangle_{L^{2}([-1,1])}=\frac{1-a^{j+1}}{j+1} \quad \text { for } j \in\{0,1\}
$$

and

$$
\left\langle\sum_{i=0}^{1} y_{i} t^{i}, t^{j}\right\rangle_{L^{2}([-1,1])} \geq\left\langle\psi_{a}, t^{j}\right\rangle_{L^{2}([-1,1])}=\frac{1-a^{j+1}}{j+1} \text { for } j \geq 2 .
$$

The equalities (4.61) can be checked directly by using the definitions of $y_{0}$ and $y_{1}$. Now let us analyze the inequalities (4.62). Note that if $j=2 n$ for $n \geq 1$, then

$$
\left\langle\sum_{i=0}^{1} y_{i} t^{i}, t^{2 n}\right\rangle_{L^{2}([-1,1])}-\left\langle\psi_{a}, t^{2 n}\right\rangle_{L^{2}([-1,1])}=\frac{2}{2 n+1} \frac{1-a}{2}-\frac{1-a^{2 n+1}}{2 n+1}=\frac{a^{2 n+1}-a}{2 n+1}
$$

and if $j=2 n+1$ with $n \geq 1$, then

$$
\begin{aligned}
\left\langle\sum_{i=0}^{1} y_{i} t^{i}, t^{2 n+1}\right\rangle_{L^{2}([-1,1])}-\left\langle\psi_{a}, t^{2 n+1}\right\rangle_{L^{2}([-1,1])} & =\frac{2}{2 n+3} \cdot \frac{3}{4}\left(1-a^{2}\right)-\frac{1-a^{2 n+2}}{2 n+2} \\
& =\frac{\left(1-3 a^{2}+2 a^{2 n+2}\right) n+3 a^{2 n+2}-3 a^{2}}{2(2 n+3)(n+1)} .
\end{aligned}
$$

Therefore, (4.62) holds if and only if

$$
\left\{\begin{array}{l}
a^{2 n+1}-a \geq 0 \\
\left(1-3 a^{2}+2 a^{2 n+2}\right) n+3 a^{2 n+2} \geq 3 a^{2}
\end{array} \text { for all integers } n \geq 1 .\right.
$$


By Lemma 4.2, under the assumption $a \in[-1,1)$, the condition (4.63) holds if and only if $-\frac{1}{\sqrt{5}} \leq a \leq 0$. This completes the proof of the proposition.

Lemmas 4.3 and 4.4 below will be used in the proof of Proposition 1.11.

Lemma 4.3. Assume that $a \in[-1,1)$. Then the solution to the linear equation

$$
M_{3} z=v_{a}^{(3)}
$$

belongs to $\left(\mathbb{R}_{+}^{*}\right)^{4}$ if and only if

$$
\frac{1}{\sqrt{5}}<a<\frac{\sqrt{105}-5}{10} .
$$

Proof. The solution $z=\left(z_{0}, z_{1}, z_{2}, z_{3}\right)$ of the linear equation (4.64) is given by

$$
\left\{\begin{array}{l}
z_{0}=\frac{1}{8}(1-a)\left(4-5 a-5 a^{2}\right) \\
z_{1}=\frac{15}{32}\left(1-a^{2}\right)\left(3-7 a^{2}\right) \\
z_{2}=\frac{15}{8} a\left(1-a^{2}\right) \\
z_{3}=\frac{35}{32}\left(1-a^{2}\right)\left(5 a^{2}-1\right)
\end{array}\right.
$$

Therefore, under the assumption $a \in[-1,1)$, the solution $z$ belongs to $\left(\mathbb{R}_{+}^{*}\right)^{4}$ if and only if $\frac{1}{\sqrt{5}}<a<\frac{\sqrt{105}-5}{10}$. This completes the proof of the lemma.

Lemma 4.4. Suppose that $\frac{1}{\sqrt{5}}<a<\frac{\sqrt{105}-5}{10}$. Then for all integers $n \geq 2$, we have

$$
\left(3 a-5 a^{3}+2 a^{2 n+1}\right) n+3 a^{2 n+1}-3 a \geq 0
$$

and

$$
\left(-3+30 a^{2}-35 a^{4}+8 a^{2 n+2}\right) n^{2}+\left(3-35 a^{4}+32 a^{2 n+2}\right) n+30 a^{2 n+2}-30 a^{2} \geq 0 .
$$

Proof. Assume that $\frac{1}{\sqrt{5}}<a<\frac{\sqrt{105}-5}{10}$. For the first inequality, note that, combined with the elementary inequality

$$
\left(\frac{\sqrt{105}-5}{10}\right)^{2}<\frac{3}{10},
$$

the assumption $\frac{1}{\sqrt{5}}<a<\frac{\sqrt{105}-5}{10}$ implies

$$
3 a-5 a^{3}=a\left(3-5 a^{2}\right)>0 \text { and } 3 a-10 a^{3}=a\left(3-10 a^{2}\right)>0 .
$$

Thus for any $n \geq 2$, we have

$$
\begin{aligned}
\left(3 a-5 a^{3}+2 a^{2 n+1}\right) n+3 a^{2 n+1}-3 a & \geq\left(3 a-5 a^{3}\right) n-3 a \\
& \geq 2\left(3 a-5 a^{3}\right)-3 a=3 a-10 a^{3}>0 .
\end{aligned}
$$

For the second inequality, note that, combined with the elementary inequality

$$
\left(\frac{\sqrt{105}-5}{10}\right)^{4}<\frac{3}{35}
$$

the assumption $\frac{1}{\sqrt{5}}<a<\frac{\sqrt{105}-5}{10}$ implies

$$
3-35 a^{4}>0
$$


and

$$
-3+30 a^{2}-35 a^{4}>-3+30\left(\frac{1}{\sqrt{5}}\right)^{2}-35 a^{4}=3-35 a^{4}>0 .
$$

Note also that

$$
-1+15 x^{2}-35 x^{4} \geq 0 \quad \text { provided that } \frac{15-\sqrt{85}}{70} \leq x^{2} \leq \frac{15+\sqrt{85}}{70} .
$$

Therefore, by noting

$$
\frac{15-\sqrt{85}}{70} \leq \frac{1}{5} \leq a^{2} \leq\left(\frac{\sqrt{105}-5}{10}\right)^{2} \leq \frac{15+\sqrt{85}}{70}
$$

we have

$$
-1+15 a^{2}-35 a^{4} \geq 0 .
$$

It follows that for any integer $n \geq 2$, we have

$$
\begin{aligned}
& \left(-3+30 a^{2}-35 a^{4}+8 a^{2 n+2}\right) n^{2}+\left(3-35 a^{4}+32 a^{2 n+2}\right) n+30 a^{2 n+2}-30 a^{2} \\
& \geq\left(-3+30 a^{2}-35 a^{4}\right) n^{2}+\left(3-35 a^{4}\right) n-30 a^{2} \\
& \geq\left(-3+30 a^{2}-35 a^{4}\right) \times 4+\left(3-35 a^{4}\right) \times 2-30 a^{2} \\
& =6\left(-1+15 a^{2}-35 a^{4}\right) \geq 0 .
\end{aligned}
$$

The lemma is proved completely.

Proof of Proposition 1.11. Let $z=\left(z_{0}, z_{1}, z_{2}, z_{3}\right)$ be given as in (4.65) and recall that $z$ is the solution to the linear equation (4.64). By the discussions in $§ 1.3$ and Proposition 1.3, the equality (1.23) holds if and only if the following conditions are all satisfied:

- $z \in\left(\mathbb{R}_{+}^{*}\right)^{4}$;

- for $j \in\{0,1,2,3\}$, we have

$$
\left\langle\sum_{i=0}^{3} z_{i} t^{i}, t^{j}\right\rangle_{L^{2}([-1,1])}=\left\langle\psi_{a}, t^{j}\right\rangle_{L^{2}([-1,1])}=\frac{1-a^{j+1}}{j+1} ;
$$

- for all integers $j \geq 4$, we have

$$
\left\langle\sum_{i=0}^{3} z_{i} t^{i}, t^{j}\right\rangle_{L^{2}([-1,1])} \geq\left\langle\psi_{a}, t^{j}\right\rangle_{L^{2}([-1,1])}=\frac{1-a^{j+1}}{j+1} .
$$

Note that the system of linear equations (4.66) for $j \in\{0,1,2,3\}$ is equivalent to the linear equation (4.64). Thus by the definition of $z$, the equalities (4.66) hold for all $j \in\{0,1,2,3\}$. By Lemma 4.3, $z \in\left(\mathbb{R}_{+}^{*}\right)^{4}$ if and only if $\frac{1}{\sqrt{5}}<a<\frac{\sqrt{105}-5}{10}$. Now let us analyze the inequalities (4.67) for $j \geq 4$. For even numbers $j=2 n$ with $n \geq 2$, we have

$$
\begin{aligned}
& \left\langle\sum_{i=0}^{3} z_{i} t^{i}, t^{2 n}\right\rangle_{L^{2}([-1,1])}-\left\langle\psi_{a}, t^{2 n}\right\rangle_{L^{2}([-1,1])} \\
& =\frac{2}{2 n+1} \cdot \frac{1}{8}(1-a)\left(4-5 a-5 a^{2}\right)+\frac{2}{2 n+3} \cdot \frac{15}{8} a\left(1-a^{2}\right)-\frac{1-a^{2 n+1}}{2 n+1} \\
& =\frac{\left(3 a-5 a^{3}+2 a^{2 n+1}\right) n+3 a^{2 n+1}-3 a}{(2 n+1)(2 n+3)}
\end{aligned}
$$


and for odd numbers $j=2 n+1$ with $n \geq 2$, we have

$$
\begin{aligned}
& \left\langle\sum_{i=0}^{3} z_{i} t^{i}, t^{2 n+1}\right\rangle_{L^{2}([-1,1])}-\left\langle\psi_{a}, t^{2 n+1}\right\rangle_{L^{2}([-1,1])} \\
& =\frac{2}{2 n+3} \cdot \frac{15}{32}\left(1-a^{2}\right)\left(3-7 a^{2}\right)+\frac{2}{2 n+5} \cdot \frac{35}{32}\left(1-a^{2}\right)\left(5 a^{2}-1\right)-\frac{1-a^{2 n+2}}{2 n+2} \\
& =\frac{\left(-3+30 a^{2}-35 a^{4}+8 a^{2 n+2}\right) n^{2}+\left(3-35 a^{4}+32 a^{2 n+2}\right) n+30 a^{2 n+2}-30 a^{2}}{2(2 n+3)(2 n+5)(2 n+2)} .
\end{aligned}
$$

Therefore, the equality (1.23) holds if and only if the following conditions are all satisfied:

- $\frac{1}{\sqrt{5}}<a<\frac{\sqrt{105}-5}{10}$

- for all integers $n \geq 2$,

$$
\left(3 a-5 a^{3}+2 a^{2 n+1}\right) n+3 a^{2 n+1}-3 a \geq 0
$$

- for all integers $n \geq 2$,

$$
\left(-3+30 a^{2}-35 a^{4}+8 a^{2 n+2}\right) n^{2}+\left(3-35 a^{4}+32 a^{2 n+2}\right) n+30 a^{2 n+2}-30 a^{2} \geq 0 .
$$

Thus by Lemma 4.4, the equality (1.23) holds if and only if $\frac{1}{\sqrt{5}}<a<\frac{\sqrt{105}-5}{10}$.

Finally, by Proposition 1.3, the above arguments imply that if $\frac{1}{\sqrt{5}}<a<\frac{\sqrt{105}-5}{10}$, then

$$
P_{\mathcal{A}_{+}}\left(\psi_{a}\right)=\sum_{i=0}^{3} z_{i} t^{i}
$$

This is the desired equality (1.24).

\section{Appendix}

In this appendix, we show that the set

$$
\mathcal{C}_{L^{2}([-1,0])}\left[\left[\left\{t^{n}\right\}_{n=0}^{\infty}\right]\right]=\left\{\sum_{n=0}^{\infty} a_{n} t^{n} \mid a_{n} \geq 0, \text { the series } \sum_{n=0}^{\infty} a_{n} t^{n} \text { converges in } L^{2}([-1,0])\right\}
$$

is not closed. Or equivalently, we show that the set

$\mathcal{C}_{L^{2}([0,1])}\left[\left[\left\{(-t)^{n}\right\}_{n=0}^{\infty}\right]\right]=\left\{\sum_{n=0}^{\infty} a_{n}(-t)^{n} \mid a_{n} \geq 0\right.$, the series $\sum_{n=0}^{\infty} a_{n}(-t)^{n}$ converges in $\left.L^{2}([0,1])\right\}$

is not closed. Indeed, set $\rho_{k}=1-\frac{1}{k+1}$ for any $k \in \mathbb{N}$. Let

$$
g_{k}(t):=\frac{1}{\left(1+\rho_{k} t\right)^{2}}=\sum_{n=0}^{\infty}(n+1) \rho_{k}^{n}(-t)^{n}, \quad t \in[0,1)
$$

Then clearly, we have $g_{k} \in \mathcal{C}_{L^{2}([0,1])}\left[\left[\left\{(-t)^{n}\right\}_{n=0}^{\infty}\right]\right]$ and

$$
g_{k}(t) \underset{k \rightarrow \infty}{\stackrel{\text { in } L^{2}([0,1])}{\longrightarrow}} g_{\infty}(t)=\frac{1}{(1+t)^{2}}
$$


Now let us show that $g_{\infty} \notin \mathcal{C}_{L^{2}([0,1])}\left[\left[\left\{(-t)^{n}\right\}_{n=0}^{\infty}\right]\right]$. Otherwise, $g_{\infty} \in \mathcal{C}_{L^{2}([0,1])}\left[\left[\left\{(-t)^{n}\right\}_{n=0}^{\infty}\right]\right]$, then there exists a sequence $\left(a_{n}\right)_{n=0}^{\infty}$ of non-negative numbers such that

$$
g_{\infty}=\sum_{n=0}^{\infty} a_{n}(-t)^{n}
$$

where the equality is understood as

$$
\lim _{N \rightarrow \infty}\left\|\sum_{n=0}^{N} a_{n}(-t)^{n}-\frac{1}{(1+t)^{2}}\right\|_{L^{2}([0,1])}=0 .
$$

The above convergence implies

$$
\frac{a_{n}^{2}}{2 n+1}=\left\|a_{n}(-t)^{n}\right\|_{L^{2}([0,1])}^{2} \leq \sup _{n}\left\|a_{n}(-t)^{n}\right\|_{L^{2}([0,1])}^{2}=M<\infty .
$$

Since the $L^{2}$-norm convergence implies the almost everywhere convergence along a subsequence, (5.68) implies that, along a subsequence $\left(N_{k}\right)_{k=0}^{\infty}$ of positive numbers, we have

$$
\lim _{k \rightarrow \infty} \sum_{n=0}^{N_{k}} a_{n}(-t)^{n}=\frac{1}{(1+t)^{2}} \quad \text { for Lebesgue a.e. } t \in[0,1] .
$$

Note that (5.69) implies that the series $\sum_{n} a_{n} z^{n}$ has a radius of convergence not smaller than 1 , hence we have

$$
\lim _{k \rightarrow \infty} \sum_{n=0}^{N_{k}} a_{n}(-t)^{n}=\sum_{n=0}^{\infty} a_{n}(-t)^{n} \quad \text { for all } t \in[0,1) .
$$

Therefore, we obtain

$$
\sum_{n=0}^{\infty} a_{n}(-t)^{n}=\frac{1}{(1+t)^{2}} \quad \text { for Lebesgue a.e. } t \in[0,1] .
$$

By elementary results on analytic funtions, the above equality implies that $a_{n}=n+1$ for all $n \in \mathbb{N}$. Thus the limit relation (5.68) now reads as

$$
\lim _{N \rightarrow \infty}\left\|\sum_{n=0}^{N}(n+1)(-t)^{n}-\frac{1}{(1+t)^{2}}\right\|_{L^{2}([0,1])}=0 .
$$

However, for any large integer $N$ and $t \in(0,1)$, we have

$$
\sum_{n=0}^{N}(n+1)(-t)^{n}-\frac{1}{(1+t)^{2}}=\sum_{n=N+1}^{\infty}(n+1)(-t)^{n}=\frac{(N+2)(-t)^{N+1}}{1+t}+\frac{(-t)^{N+2}}{(1+t)^{2}}
$$

Since

$$
\left\|\frac{(N+2)(-t)^{N+1}}{1+t}\right\|_{L^{2}([0,1])} \geq \frac{N+2}{2}\left\|(-t)^{N+1}\right\|_{L^{2}([0,1])}=\frac{N+2}{2 \sqrt{2 N+3}}
$$

and

$$
\left\|\frac{(-t)^{N+2}}{(1+t)^{2}}\right\|_{L^{2}([0,1])} \leq 1
$$


we have

$$
\lim _{N \rightarrow \infty}\left\|\sum_{n=0}^{N}(n+1)(-t)^{n}-\frac{1}{(1+t)^{2}}\right\|_{L^{2}([0,1])} \geq \lim _{N \rightarrow \infty}\left(\frac{N+2}{2 \sqrt{2 N+3}}-1\right)=\infty .
$$

Thus we obtain a contradiction. Hence $\mathcal{C}_{L^{2}([0,1])}\left[\left[\left\{(-t)^{n}\right\}_{n=0}^{\infty}\right]\right]$ is not closed in $L^{2}([0,1])$.

\section{REFERENCES}

[1] L. Asimow and A. J. Ellis. Convexity theory and its applications in functional analysis, volume 16 of London Mathematical Society Monographs. Academic Press, Inc. [Harcourt Brace Jovanovich, Publishers], London-New York, 1980.

[2] A. Beck and N. Hallak. On the convergence to stationary points of deterministic and randomized feasible descent directions methods. SIAM J. Optim., 30(1):56-79, 2020.

[3] J. M. Borwein and A. S. Lewis. Convex analysis and nonlinear optimization, volume 3 of CMS Books in Mathematics/Ouvrages de Mathématiques de la SMC. Springer, New York, second edition, 2006. Theory and examples.

[4] C. Davis. Theory of positive linear dependence. Amer. J. Math., 76:733-746, 1954.

[5] F. Deutsch. Best approximation in inner product spaces, volume 7 of CMS Books in Mathematics/Ouvrages de Mathématiques de la SMC. Springer-Verlag, New York, 2001.

[6] F. Deutsch, J. H. McCabe and G. M. Phillips. Some algorithms for computing best approximations from convex cones. SIAM J. Numer.Anal. 12 (1975), 390403.

[7] A. Domokos, J. M. Ingram and M. M. Marsh. Projections onto closed convex sets in Hilbert spaces. Acta Math. Hungar., 152(1):114-129, 2017.

[8] R. E. Edwards. Functional analysis. Theory and applications. Holt, Rinehart and Winston, New York-Toronto-London, 1965.

[9] J. M. Ingram and M. M. Marsh. Projections onto convex cones in Hilbert space. J. Approx. Theory, 64(3):343-350, 1991.

[10] W. B. Jurkat and G. G. Lorentz. Uniform approximation by polynomials with positive coefficients. Duke Math. J., 28:463-473, 1961.

[11] R. L. McKinney. Positive bases for linear spaces. Trans. Amer. Math. Soc., 103:131-148, 1962.

[12] R. D. Nussbaum and B. Walsh. Approximation by polynomials with nonnegative coefficients and the spectral theory of positive operators. Trans. Amer. Math. Soc., 350(6):2367-2391, 1998.

[13] J. Stoer and C. Witzgall. Convexity and optimization in finite dimensions. I. Die Grundlehren der mathematischen Wissenschaften, Band 163. Springer-Verlag, New York-Berlin, 1970.

[14] J. F. Toland. Self-adjoint operators and cones. J. London Math. Soc. (2), 53(1):167-183, 1996.

[15] D. E. Wulbert. Continuity of metric projections. Trans. Amer. Math. Soc., 134:335-341, 1968.

[16] E. H. Zarantonello. Projections on convex sets in Hilbert space and spectral theory. I. Projections on convex sets. In Contributions to nonlinear functional analysis (Proc. Sympos., Math. Res. Center, Univ. Wisconsin, Madison, Wis., 1971), pages 237-341, 1971.

Yanqi QIU: Institute of Mathematics and Hua Loo-Keng Key Laboratory of Mathematics, AMSS, Chinese Academy of Sciences, Beijing 100190, China.

E-mail address: yanqi.qiulamss.ac.cn, yanqi.qiu@hotmail.com

Zipeng WANG: College of Mathematics and Statistics, Chongqing University, Chongqing, 401331, P.R.CHINA

E-mail address: zipengwang2012@gmail.com, zipengwang@cqu.edu.cn 